Supporting Information for:

\title{
In vitro biosynthesis of the core scaffold of the thiopeptide thiomuracin
}

Graham A. Hudson, ${ }^{\dagger, \perp}$ Zhengan Zhang, ${ }^{\dagger, \perp}$ Jonathan I. Tietz, ${ }^{\dagger}$ Douglas A. Mitchell, ${ }^{\dagger,} *$ and Wilfred A. van der Donk, ${ }^{\dagger, *} *$

'Department of Chemistry, ${ }^{\dagger}$ Carl R. Woese Institute for Genomic Biology University of Illinois at UrbanaChampaign, Urbana, Illinois, 61801 , USA. ${ }^{\perp}$ These authors contributed equally.

* Corresponding authors:

Wilfred A. van der Donk (vddonk@illinois.edu), phone: 1-217-244-5360, fax: 1-217-244-8533

Douglas A. Mitchell (douglasm@illinois.edu), phone: 1-217-333-1345, fax: 1-217-333-0508

\section{Table of Contents:}

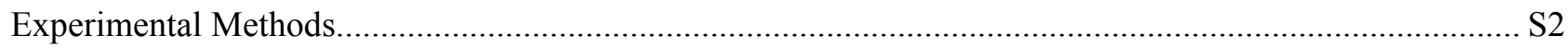

Table S1: Sequence of $t b t$ biosynthetic genes for optimal E. coli expression ............................................ S7

Table S2: Oligonucleotide primers used in this study ........................................................................... S11

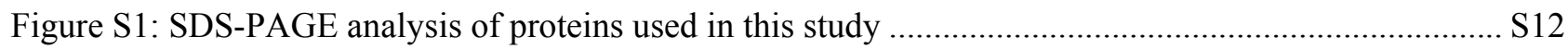

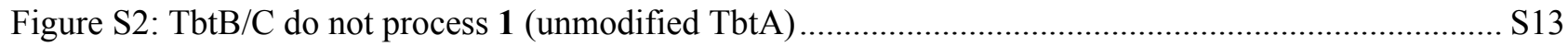

Figure S3: MALDI-MS analysis of TbtE/F/G reaction requirements ................................................. S14

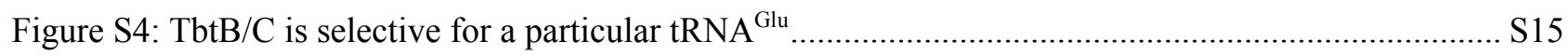

Figure S5: MALDI-MS analysis of TbtB/C reaction requirements ......................................................... S16

Figure S6: TbtD requires the leader peptide for activity .................................................................. S17

Figure S7: TbtD activity is not enhanced by known leader peptide-binding Tbt proteins ........................ S18

Figure S8: Purity analysis of 4 (macrocylized, thiomuracin GZ product)............................................... S19

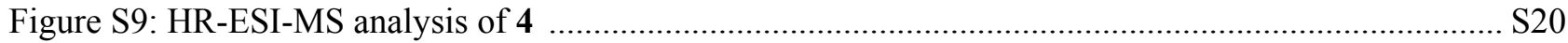

Figure S10: Structurally overlaid assignments for atom numbering and ${ }^{1} \mathrm{H} /{ }^{13} \mathrm{C}-\mathrm{NMR}$ chemical shifts of $4 \mathrm{~S} 21$

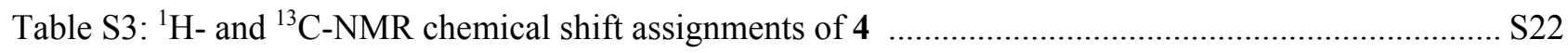

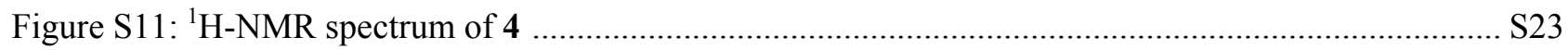

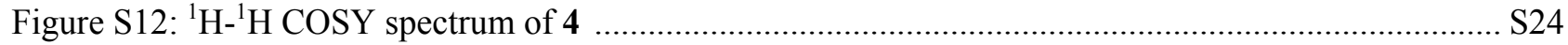

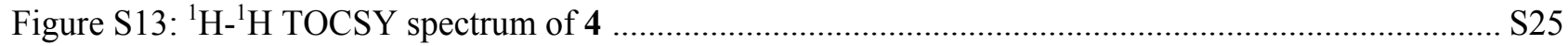

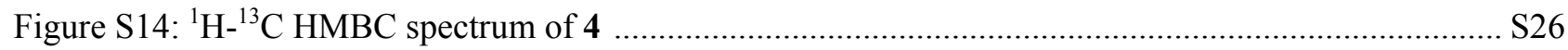

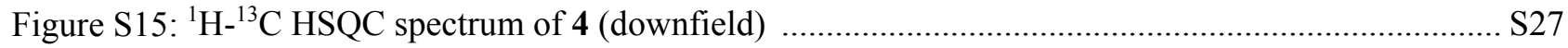

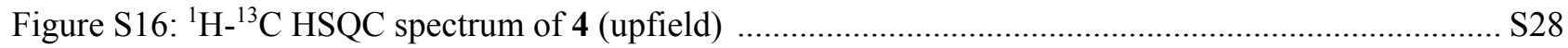

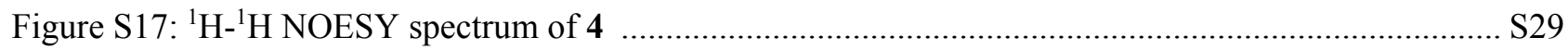

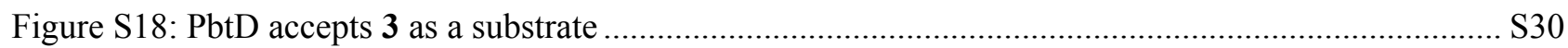

Figure S19: Sequence alignment of alanine-substituted TbtD proteins ................................................ S31

Table S4: Yield and activity of alanine-substituted TbtD proteins …................................................... S32

Table S5: Enzymatic activity of selected TbtD mutant proteins .......................................................... S33

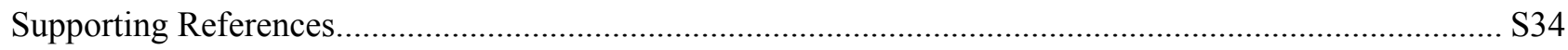




\section{Experimental Methods.}

General materials and methods. Reagents used for molecular biology experiments were purchased from New England BioLabs (Ipswich, MA), Thermo Fisher Scientific (Waltham, MA), or Gold Biotechnology Inc. (St. Louis, MO). Other chemicals were purchased from Sigma-Aldrich (St. Louis, MO). Escherichia coli DH5 $\alpha$ and BL21 (DE3) strains were used for plasmid maintenance and protein overexpression, respectively. Thermobispora bispora DSM 43833 was obtained from DSMZ (Braunschweig, Germany). Planobispora rosea ATCC53733 was obtained from the USDA Agricultural Research Service (ARS) Culture Collection. Plasmid inserts were sequenced at ACGT Inc. (Wheeling, IL). MALDI-TOF-MS analysis was performed using a Bruker UltrafleXtreme MALDI TOF-TOF mass spectrometer (Bruker Daltonics) in reflector positive mode at the University of Illinois School of Chemical Sciences Mass Spectrometry Laboratory. ESI-MS/MS analyses were performed using a SYNAPT ESI quadrupole TOF Mass Spectrometry System (Waters) equipped with an ACQUITY Ultra Performance Liquid Chromatography (UPLC) system (Waters). Kinetic assays were performed using a Varian Cary $4000 \mathrm{UV}$-Vis spectrophotometer. HiTrap columns for Ni-NTA affinity chromatography were purchased from GE Healthcare.

Molecular biology techniques. Oligonucleotides were purchased from Integrated DNA Technologies Inc. (Coralville, IA). Primers for E. coli $\mathrm{tRNA}^{\text {Glu }}$ were designed and the dsDNA template for in vitro transcription was prepared as previously described. ${ }^{1}$ Thermobispora bispora was identified as a potential producer of a thiomuracinlike thiopeptide using BLAST to identify homologs of genes from the relevant biosynthetic gene cluster of thiomuracin producer Nonomurea sp. Bp3714-39. ${ }^{2}$ Genes optimized for recombinant expression in Escherichia coli were synthesized by GenScript (Piscataway, NJ) in pUC57 (kanamycin, Kan) vectors with BamHI and XhoI sites flanking each gene at the $5^{\prime}$ and $3^{\prime}$ ends, respectively. The GenBank locus tag and E. coli optimized sequence for each gene is provided in Table S1. E. coli DH5 $\alpha$ were transformed with pUC57-Kan vectors containing each gene for replication and subsequent isolation using a QIAprep Spin Miniprep Kit (Qiagen). The isolated DNA was then treated with BamHI-HF and XhoI-HF (New England Biolabs). The digested genes were separated on a 1\% (w/v) agarose gel, purified using a QIAQuick gel extraction kit (Qiagen), and ligated into an appropriately endonucleasedigested and gel-purified pET28 vectors using T4 DNA ligase (NEB). Ligation reactions were used to transform chemically competent DH5 $\alpha$ cells, which were plated on Luria-Bertani (LB) agar plates containing $50 \mu \mathrm{g} / \mathrm{mL}$ kanamycin and grown at $37^{\circ} \mathrm{C}$. Colonies were picked at random and grown in LB broth for 16-20 h prior to plasmid isolation using a QIAprep Spin Miniprep Kit. For TbtA and TbtD, the vector encoded a tobacco etch virus (TEV) cleavable, N-terminal maltose binding protein (MBP) affinity tag, while TbtE, TbtF, and TbtG constructs did not encode a TEV proteolysis site. All recombinant constructs featuring an MBP-tag were sequenced using a custom MBP forward primer, whereas constructs encoding His 6 -tagged proteins were sequenced using the T7 forward primer. All constructs were reverse- sequenced using a T7 reverse primer as well as an internal primer if necessary (Table S2).

The gene encoding PbtD (TbtD ortholog) from Planobispora rosea ATCC 23866 was amplified from genomic DNA using the primers listed in Table S2. The resulting DNA was purified using a QIAprep Spin Miniprep Kit. The purified DNA was then digested using BamHI-HF and XhoI-HF before being ligated into an appropriately digested pET28 vector encoding an N-terminal MBP tag featuring a TEV proteolysis site. The resulting construct was then used to transform E. coli, propagated, and sequenced as described above.

Site-directed mutagenesis on TbtD was performed using the QuikChange method (Agilent) as per the manufacturer's instructions using PfuTurbo DNA polymerase. The primers for each mutant are listed in Table S2. The mutations were verified by sequencing using the MBP forward primer or the $\mathrm{T} 7$ reverse primer.

The tbtA gene was cloned into $p R S F D u e t-1$ with an $N$-terminal His 6 -tag following standard cloning procedures. In addition, the GlySerSer residues preceding the $\mathrm{His}_{6}$ tag were mutated to a single Pro to suppress gluconoylation. ${ }^{3}$ The PCR product was purified by gel extraction on a $1 \%(\mathrm{w} / \mathrm{v})$ agarose gel using the QIAquick Gel Extraction Kit (Qiagen). The vector $p R S F D u e t-1$ was digested using BamHI-HF and HindIII-HF (NEB) restriction endonucleases and purified by gel extraction as described above. Insertion of the DNA fragment was achieved by Gibson one-step isothermal DNA assembly as previously described. ${ }^{4}$ An aliquot $(20 \mu \mathrm{L})$ of the Gibson assembly reaction was used to transform $E$. coli $\mathrm{DH} 5 \alpha$ cells using the heat shock method. The cells were plated on LB plates supplemented with kanamycin $(50 \mu \mathrm{g} / \mathrm{mL})$ and single colonies were grown at $37^{\circ} \mathrm{C}$ for $12-15 \mathrm{~h}$. The plasmid His $\sigma^{-T b t A}-$ pRSFDuet- 1 
was isolated using a QIA Spin Miniprep Kit. Insert integrity was verified by sequencing the plasmids with the appropriate primers (Table $\mathbf{S 2}$ ).

The gene coding for the dehydratase TbtB was cloned into $p E T 28 a$ encoding an N-terminal His 6 -tag. The $t b t B$ gene was amplified by PCR, and purified by gel extraction from a $1 \%(\mathrm{w} / \mathrm{v})$ agarose gel using the QIAquick Gel Extraction Kit. The vector $p E T 28 a$ and the $t b t B$ PCR fragment were digested using BamHI-HF and XhoI-HF (NEB) restriction endonucleases, and purified by gel extraction as described above. The DNA fragment was inserted by Gibson one-step isothermal DNA assembly, and an aliquot of $20 \mu \mathrm{L}$ of the Gibson assembly reaction was used to transform E. coli DH5 $\alpha$ cells using the heat shock method. The cells were plated on LB plates supplemented with kanamycin $(50 \mu \mathrm{g} / \mathrm{mL})$ and the plates were incubated at $37^{\circ} \mathrm{C}$ for $12-15 \mathrm{~h}$. Single colonies were picked and grown in LB supplemented with kanamycin $(50 \mu \mathrm{g} / \mathrm{mL})$ at $37^{\circ} \mathrm{C}$ for $12-15 \mathrm{~h}$ and the plasmid His ${ }_{6}$ TbtB-pET28a was isolated using a QIA prep Spin Miniprep Kit. Insert integrity was verified by sequencing the plasmids with the appropriate primers (Table S2). All the other genes and their corresponding His ${ }_{6}$-tagged proteins were cloned using the same procedures.

MBP-tagged peptide and enzyme overexpression and purification. E. coli BL21(DE3) cells were transformed with a pET28 plasmid encoding the MBP-tagged enzyme or peptide of interest. Cells were grown for $24 \mathrm{~h}$ on LB agar plates containing $50 \mu \mathrm{g} / \mathrm{mL}$ kanamycin at $37^{\circ} \mathrm{C}$. Single colonies were used to inoculate $10 \mathrm{~mL}$ of LB containing $50 \mu \mathrm{g} / \mathrm{mL}$ kanamycin and grown at $37^{\circ} \mathrm{C}$ for $16-18 \mathrm{~h}$. This culture was used to inoculate $1 \mathrm{~L}$ of Terrific Broth $(24$ $\mathrm{g} / \mathrm{L}$ yeast extract, $12 \mathrm{~g} / \mathrm{L}$ tryptone, $0.4 \%$ glycerol (v/v), $17 \mathrm{mM} \mathrm{KH}_{2} \mathrm{PO}_{4}$, and $72 \mathrm{mM} \mathrm{K}_{2} \mathrm{HPO}_{4}$ ) and grown to an optical density at $600 \mathrm{~nm}\left(\mathrm{OD}_{600}\right)$ of 1.0 before being placed on ice for $15 \mathrm{~min}$. Protein expression was then induced with the addition of $0.4 \mathrm{mM}$ isopropyl $\beta$-D-1-thiogalactopyranoside (IPTG) for biosynthetic enzymes or $1 \mathrm{mM}$ IPTG for the TbtA precursor peptide. Expression was allowed to proceed for $16-18 \mathrm{~h}$ at $22{ }^{\circ} \mathrm{C}$. For expression of MBP-TbtE and MBP-TbtG, $70 \mu \mathrm{M}$ riboflavin or $100 \mu \mathrm{M} \mathrm{ZnCl}_{2}$, respectively, were added at the time of induction. Cells were harvested by centrifugation at 3,000 $\times \mathrm{g}$ for $20 \mathrm{~min}$, washed with phosphate-buffered saline (PBS; 137 $\mathrm{mM} \mathrm{NaCl}, 2.7 \mathrm{mM} \mathrm{KCl}, 10 \mathrm{mM} \mathrm{Na}_{2} \mathrm{HPO}_{4}$, and $1.8 \mathrm{mM} \mathrm{KH}_{2} \mathrm{PO}_{4}$ ), and centrifuged again. The cell pellet was flashfrozen and stored at $-80{ }^{\circ} \mathrm{C}$ for a maximum of one week before use.

Cell pellets were resuspended in lysis buffer $(50 \mathrm{mM}$ Tris- $\mathrm{HCl} \mathrm{pH} 7.5,150 \mathrm{mM} \mathrm{NaCl}, 2.5 \%$ glycerol (v/v), and $0.1 \%$ Triton X-100) containing $4 \mathrm{mg} / \mathrm{mL}$ lysozyme, $2 \mu \mathrm{M}$ leupeptin, $2 \mu \mathrm{M}$ benzamidine, and $2 \mu \mathrm{M}$ E64. After 30 min at $4{ }^{\circ} \mathrm{C}$, cells were further homogenized by sonication $\left(3 \times 45 \mathrm{~s}\right.$ with 10 min nutation periods at $\left.4{ }^{\circ} \mathrm{C}\right)$. Insoluble debris was removed by centrifugation at $20,000 \times g$ for $90 \mathrm{~min}$. The supernatant was then applied to a preequilibrated amylose resin (NEB; $20 \mathrm{~mL}$ of resin per L of cells). The column was washed with 10 column volumes (CV) of lysis buffer followed by $10 \mathrm{CV}$ of wash buffer (lysis buffer with $400 \mathrm{mM} \mathrm{NaCl}$ and lacking Triton X-100). The MBP-tagged proteins were eluted using elution buffer (lysis buffer with $300 \mathrm{mM} \mathrm{NaCl}, 10 \mathrm{mM}$ maltose, and also lacking Triton X-100) until the eluent no longer contained protein detectable with the Bradford reagent (assayed using $2 \mu \mathrm{L}$ of eluent and $198 \mu \mathrm{L}$ of Bradford solution at rt). Eluent was concentrated using an appropriate MWCO Amicon Ultra centrifugal filter (EMD Millipore). A buffer exchange with $10 \times$ volume of protein storage buffer (50 $\mathrm{mM}$ HEPES $\mathrm{pH} 7.5,300 \mathrm{mM} \mathrm{NaCl}, 2.5 \%$ glycerol $(\mathrm{v} / \mathrm{v}))$ was performed prior to final concentration and storage. Protein concentrations were assayed using both $280 \mathrm{~nm}$ absorbance (theoretical extinction coefficients were calculated using the ExPASy ProtParam tool; http://web.expasy.org/protparam/protpar-ref.html) and a Bradford colorimetric assay. Purity and possible truncation were assessed by Coomassie-stained SDS-PAGE gel (Figure S1). All wash, elution, and storage buffers were supplemented with $0.5 \mathrm{mM}$ tris-(2-carboxyethyl)-phosphine (TCEP) except for those used for the preparation of MBP-TbtD (to avoid nucleophilic addition of TCEP to the dehydroalanine residues).

Expression, and purification of His6-TbtA (1). E. coli BL21 (DE3) cells (50 $\mu \mathrm{L})$ were electroporated with His $\sigma^{-}$ TbtA-pRSFDuet-1 (50 ng), plated on LB agar plates supplemented with kanamycin (as above) and grown at $37^{\circ} \mathrm{C}$ for 12-15 h. A single colony was used to inoculate $60 \mathrm{~mL}$ of LB broth supplemented with kanamycin, grown for $12-15 \mathrm{~h}$ at $37^{\circ} \mathrm{C}$, and the culture was used to inoculate $6 \mathrm{~L}$ of LB media, supplemented with kanamycin, to an $\mathrm{OD}_{600}$ of 0.025 . Cultures were grown at $37{ }^{\circ} \mathrm{C}$ to an $\mathrm{OD}_{600}$ of 1.0. Peptide expression was induced by the addition of IPTG to a final concentration of $1 \mathrm{mM}$ and cultures were grown at $37^{\circ} \mathrm{C}$ for $3 \mathrm{~h}$. Peptide purification was performed following a previously described method. ${ }^{5}$ 
Expression, and purification of His $_{6}$-TbtB. E. coli BL21 (DE3) cells $(50 \mu \mathrm{L})$ were electroporated with $\mathrm{His}_{6}$-TbtBpET28a (50 ng), and cells were plated on LB agar plates supplemented with kanamycin and grown at $37^{\circ} \mathrm{C}$ for $12-$ $15 \mathrm{~h}$. A single colony was used to inoculate $20 \mathrm{~mL}$ of LB broth supplemented with kanamycin, grown for $12-15 \mathrm{~h}$ at $37^{\circ} \mathrm{C}$, and the culture was used to inoculate $2 \mathrm{~L}$ of TB media, supplemented with kanamycin, to an $\mathrm{OD}_{600}$ of 0.025 . Cultures were grown at $37^{\circ} \mathrm{C}$ to a final $\mathrm{OD}_{600}$ of $0.6-0.8$. Protein overexpression was induced by the addition of IPTG to a final concentration of $0.2 \mathrm{mM}$, and cultures were grown at $18{ }^{\circ} \mathrm{C}$ for $18 \mathrm{~h}$. Protein purification was performed following a similar protocol as described before. ${ }^{6}$ All other His 6 -tagged proteins were purified using the same procedure.

Cyclodehydration of TbtA precursor peptide. Thiazole installation was carried out with $50 \mu \mathrm{M}$ MBP-tagged TbtA and $1.5 \mu \mathrm{M}$ of MBP-tagged biosynthetic enzymes (TbtE, TbtF, and TbtG) in synthetase buffer [50 mM Tris $\mathrm{pH} 8.0,125 \mathrm{mM} \mathrm{NaCl}, 2 \mathrm{mM}$ TCEP, $20 \mathrm{mM} \mathrm{MgCl}_{2}$, and $6 \mathrm{mM} \mathrm{ATP]}$. The MBP-tag on the precursor peptide was removed using $250 \mu \mathrm{M}$ TEV protease, and reactions were allowed to proceed for $3 \mathrm{~h}$ at $25^{\circ} \mathrm{C}$; the MBP-tagged biosynthetic enzymes did not contain a TEV cleavage site. Aliquots of $50 \mu \mathrm{L}$ were desalted via C18 ZipTip (EMD Millipore) according to the manufacturer's instructions, and the peptide was eluted using a saturated solution of sinapinic acid in 50\% aq. MeCN. The reaction was checked for completion by MALDI-TOF MS. The depletion of unmodified peptide and the appearance of the hexazole species were indicative of reaction completion.

Purification of 2. The cyclodehydration/dehydrogenation reaction mixtures were applied to Ni-NTA resin (Qiagen) equilibrated with $50 \mathrm{mM}$ Tris- $\mathrm{HCl} \mathrm{pH} 8.0,150 \mathrm{mM} \mathrm{NaCl}$ using $1 \mathrm{~mL}$ resin per $1 \mathrm{~mL}$ of reaction. The flow-through was collected, and the column was subsequently washed with $5 \mathrm{~mL}$ of wash buffer $(50 \mathrm{mM}$ Tris- $\mathrm{HCl} \mathrm{pH} 8.0,1 \mathrm{M}$ $\mathrm{NaCl}, 75 \mathrm{mM}$ imidazole) per $1 \mathrm{~mL}$ of reaction mixture. The total volume was then applied to a Thermo C18 HyperSep cartridge equilibrated first with $10 \mathrm{CV}$ of $\mathrm{MeCN}$ followed by $10 \mathrm{CV}$ of $5 \%$ aq. MeCN containing $0.3 \%$ formic acid. The column was washed with $10 \mathrm{CV}$ of $5 \%$ aq. MeCN containing $0.3 \%$ formic acid to remove polar contaminants. 2 was eluted from the column using up to $5 \mathrm{CV}$ of $80 \%$ aq. MeCN. From a reaction using $26.5 \mathrm{mg}$ TEV-cleaved TbtA, $20.0 \mathrm{mg}$ of crude 2 was isolated (77\% yield).

In vitro transcription of $\boldsymbol{T}$. bispora $\mathrm{tRNA}^{\mathrm{Glu}}$ (CUC). Primers for $T$. bispora $\mathrm{tRNA}{ }^{\mathrm{Glu}}$ were designed according to a previously described method. ${ }^{1}$ The tRNA ${ }^{\text {Glu }}$ dsDNA template was generated from two overlapping synthetic deoxyoligonucleotides. To prepare the dsDNA template for in vitro transcription, $5^{\prime}$ overhangs were filled in using the following conditions: NEB Buffer $2(1 \times)$, primers $(4 \mu \mathrm{M}$ each $)$, dNTP $(100 \mu \mathrm{M}$ each), DNA polymerase I large (Klenow) fragment $\left(1 \mathrm{U} \mathrm{\mu g}^{-1} \mathrm{DNA}\right)$ in a final volume of $50 \mu \mathrm{L}$. The reaction was incubated at $25{ }^{\circ} \mathrm{C}$ for $15 \mathrm{~min}$, quenched with EDTA $(10 \mathrm{mM})$ at $75^{\circ} \mathrm{C}$ for $25 \mathrm{~min}$, and dsDNA tRNA ${ }^{\text {Glu }}$ template was precipitated with cold EtOH overnight. In vitro transcription was performed using a previously described method. ${ }^{7}$ The transcribed tRNA ${ }^{\text {Glu }}$ was then purified by acidic phenol extraction using a previously described method. ${ }^{8}$

In vitro dehydration of 2. The following reaction conditions were used for dehydration assays: HEPES pH 7.5 (100 mM), $\mathrm{MgCl}_{2}(5 \mathrm{mM}), \mathrm{KCl}(150 \mathrm{mM})$, glutamate $(10 \mathrm{mM})$, ATP $(5 \mathrm{mM})$, TbtA hexazole $(50 \mu \mathrm{M})$, T. bispora tRNA $^{\text {Glu }}(10 \mu \mathrm{M})$, E coli GluRS $(10 \mu \mathrm{M})$, TbtB $(5 \mu \mathrm{M})$, TbtC $(5 \mu \mathrm{M})$, and thermostable inorganic pyrophosphatase

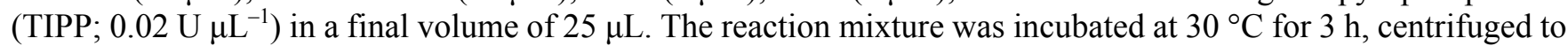
remove insoluble material $\left(14,000 \times \mathrm{g}, 5 \mathrm{~min}, 25^{\circ} \mathrm{C}\right.$ ), and desalted using C18 ZipTips (EMD Millipore). The sample was mixed in a 1:1 ratio with 2,5-dihydroxybenzoic acid matrix, spotted on a Bruker MALDI plate, and analyzed by MALDI-TOF-MS.

Purification of 3. The reaction mixtures to produce 3 were lyophilized, and the residue was extracted with a volume of $40 \%$ aq. MeCN equal to the volume used for the dehydration reaction. The insoluble material was removed by centrifugation at $10,000 \times g$ for $10 \mathrm{~min}$, and the supernatant was purified on a Shimadzu Prominence Preparative Liquid Chromatography system equipped with a Phenomenex Luna C18 column $(250 \times 10 \mathrm{~mm}, 10 \mu \mathrm{m}$ particle size, $100 \AA$ pore size). Acetonitrile and $10 \mathrm{mM}$ aq. $\mathrm{NH}_{4} \mathrm{HCO}_{3}$ were used as the mobile phases, and a gradient of 2$80 \%$ aq. MeCN over $45 \mathrm{~min}$ at $1 \mathrm{~mL} / \mathrm{min}$ was used for separation.

Purification of TbtA-hexazole-tetradehydrate core. HPLC-purified TbtA-hexazole-tetradehydrate was dissolved in $50 \mathrm{mM}$ Tris- $\mathrm{HCl}(\mathrm{pH} 7.5)$ to a final concentration of $100 \mu \mathrm{M}$. GluC endoproteinase was added to a concentration of $0.02 \mathrm{mg} / \mathrm{mL}$, and the reaction mixture was incubated at $37{ }^{\circ} \mathrm{C}$ for $1 \mathrm{~h}$, followed by addition of $\mathrm{MeCN}$ to $50 \%$ 
$(\mathrm{v} / \mathrm{v})$ to precipitate proteins. The insoluble material was removed by centrifugation at $16,100 \times \mathrm{g}$ for $10 \mathrm{~min}$, and the supernatant was purified on an Agilent 1260 Infinity HPLC system with a Phenomenex Luna C18 column (250 $\times 4.6 \mathrm{~mm}, 10 \mu \mathrm{m}$ particle size, $100 \AA$ pore size). Acetonitrile and $10 \mathrm{mM} \mathrm{NH}_{4} \mathrm{HCO}_{3}$ were used as the mobile phases, and a gradient of $2-80 \%$ aq. MeCN over $45 \mathrm{~min}$ at $1 \mathrm{~mL} / \mathrm{min}$ was used for separation.

In vitro production of 4 . Purified 2 was dissolved in DMSO to a concentration of $1 \mathrm{mM}$. The in vitro production of macrocycle was performed using: HEPES pH $7.5(100 \mathrm{mM}), \mathrm{MgCl}_{2}(5 \mathrm{mM}), \mathrm{KCl}(150 \mathrm{mM}), \mathrm{ATP}(5 \mathrm{mM}), 2$ $(100 \mu \mathrm{M})$, T. bispora tRNA ${ }^{\text {Glu }}(10 \mu \mathrm{M})$, E. coli GluRS $(10 \mu \mathrm{M})$, TbtB $(5 \mu \mathrm{M})$, TbtC $(5 \mu \mathrm{M})$, MBP-TbtD $(5 \mu \mathrm{M})$,

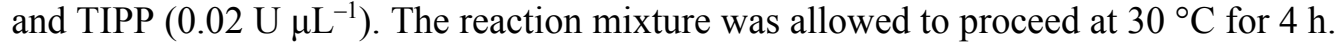

Purification of 4 (thiomuracin GZ). Reaction mixtures containing crude 4 were lyophilized and extracted with an equal volume of dimethylformamide (DMF) with respect to the original volume of the reaction by heating to $50{ }^{\circ} \mathrm{C}$ with occasional agitation for $30 \mathrm{~min}$. The insoluble material was removed by centrifugation at $18,000 \times g$ for 15 min. The supernatant was removed and evaporated in a Thermo Scientific Savant SpeedVac system. The resulting material was resuspended in $80 \%$ aq. MeCN prior to 3:1 dilution with $\mathrm{H}_{2} \mathrm{O}$. The dissolved material was then purified using a Flexar HPLC system (PerkinElmer) equipped with a Betasil C18 column $(4.6 \times 250 \mathrm{~mm}, 5 \mu \mathrm{m}$ particle size, $100 \AA$ pore size; Thermo Scientific) operating at $1 \mathrm{~mL} / \mathrm{min}$. Following a 10 minute hold at $20 \%$ aq. MeCN, separation was achieved with a gradient of $20-90 \%$ aq. MeCN over 45 min and monitoring at the $\lambda_{\max }$ for thiomuracin GZ of $315 \mathrm{~nm}$. Collected fractions were confirmed to contain thiomuracin GZ through MALDI-TOF MS $(m / z 1366 \mathrm{Da})$ analysis as previously described. The appropriate fractions were pooled and dried using the SpeedVac evaporator to yield purified thiomuracin GZ. A reaction using $20.0 \mathrm{mg} 2$ yielded $1.3 \mathrm{mg}$ of purified thiomuracin GZ, representing a yield of $27 \%$ for this step and an overall unoptimized yield of $21 \%$ for all biosynthetic steps.

NMR spectroscopy. Samples were prepared by dissolving ca. $1.1 \mathrm{mg}$ of thiomuracin GZ (HPLC-purified and lyophilized) in $180 \mu \mathrm{L}$ of DMSO- $d_{6}$ (99.96 atom \% D; Sigma-Aldrich). NMR spectra were obtained on a Bruker $900 \mathrm{MHz}$ AVANCE NMR spectrometer equipped with an inverse $5 \mathrm{~mm}$ TCI cryogenic probe with z-axis pulsed field gradient (pfg) capability. Samples were held at $298 \mathrm{~K}$ during acquisition. Standard Bruker pulse sequences were used for each of the following experiments: ${ }^{1} \mathrm{H},{ }^{1} \mathrm{H}-{ }^{1} \mathrm{H}$ COSY,${ }^{1} \mathrm{H}-{ }^{1} \mathrm{H}$ TOCSY $\left(60 \mathrm{~ms}\right.$ mixing time), ${ }^{1} \mathrm{H}-{ }^{13} \mathrm{C}$ $\mathrm{HSQC},{ }^{1} \mathrm{H}-{ }^{13} \mathrm{C} \mathrm{HMBC}$ (optimized for $6 \mathrm{~Hz}$ heteronuclear couplings), and ${ }^{1} \mathrm{H}-{ }^{1} \mathrm{H}$ NOESY (750 ms mixing time). Spectra were recorded with Bruker TopSpin 1.3 software, and data was processed using MestReNova 8.1.1. Chemical shifts $(\delta, \mathrm{ppm})$ were referenced internally to the solvent peak (DMSO).

Evaluation of thiomuracin GZ antibiotic activity. Escherichia coli MC4100, Pseudomonas aeuroginosa PA01, Bacillus anthracis strain Sterne, Mycobacterium smegmatis B-14616, Enterococcus faecium U503, and Staphylococcus aureus USA300 were grown in $10 \mathrm{~mL}$ of brain-heart infusion (BHI) medium at $37^{\circ} \mathrm{C}$. The cultures were adjusted to an $\mathrm{OD}_{600}$ of 0.015 in the designated medium before being added to 96-well microplates. Successive two-fold dilutions of thiomuracin GZ in DMSO were added to the cultures $(0.03-64 \mu \mathrm{g} / \mathrm{mL})$. As a positive control, kanamycin $(1-32 \mu \mathrm{g} / \mathrm{mL})$ was added to samples of E. coli, B. anthracis, and P. aeruginosa. Gentamicin was used a positive control for $S$. aureus and $E$. faecium while streptomycin was used for M. smegmatis. As a negative control, $1 \%(\mathrm{v} / \mathrm{v})$ DMSO was added to samples of each strain. Plates were covered and incubated at $37{ }^{\circ} \mathrm{C}$ for $20 \mathrm{~h}$ with shaking at $180 \mathrm{rpm}$. The minimum inhibitory concentration (MIC) reported are the values which suppressed all visible growth $(n=3)$.

Evaluation of thiomuracin GZ antifungal activity. Saccharomyces cerevisiae, Talaromyces stipitatus, and Aspergillus niger were grown for $36 \mathrm{~h}$ in $6 \mathrm{~mL}$ of YPD medium $(10 \mathrm{~g} / \mathrm{L}$ yeast extract, $20 \mathrm{~g} / \mathrm{L}$ peptone, and $20 \mathrm{~g} / \mathrm{L}$ dextrose) at $30^{\circ} \mathrm{C}$. S. cerevisiae cultures were diluted to $\mathrm{OD}_{600}$ of 0.015 in YPD medium before being added to 96well microplates. T. stipitatus and A. niger were not diluted prior to distribution on 96-well microplates. Successive two-fold dilutions of thiomuracin GZ in DMSO were added to cultures (1-128 $\mu \mathrm{g} / \mathrm{mL})$. As a positive control, amphotericin B was added to cultures in dilutions of $0.5-8 \mu \mathrm{g} / \mathrm{mL}$. As a negative control, $1 \%$ DMSO (v/v) was added to samples of each fungal species. Plates were covered and incubated at $30{ }^{\circ} \mathrm{C}$ for $36 \mathrm{~h}$ with shaking at 180 $\mathrm{rpm}$. The reported MICs are the values which suppressed all visible growth $(n=3)$. 
UPLC-MS of purified thiomuracin GZ. Purified thiomuracin GZ was diluted in $20 \%$ aq. MeCN to $10 \mu \mathrm{M}$, and LC-MS was performed using a Waters SYNAPT mass spectrometer outfitted with an ACQUITY UPLC, an ACQUITY Bridged Ethyl Hybrid C8 column $(2.1 \times 50 \mathrm{~mm}, 1.7 \mu \mathrm{m}$ particle size, $200 \AA \AA$; Waters $)$, an ESI ion source, and a quadruple TOF detector. A gradient of $10-90 \%$ aq. MeCN with $0.1 \%$ formic acid (v/v) over 15 min was used, and the total ion chromatogram (TIC) was recorded (Figure S8).

HPLC of purified thiomuracin GZ. $50 \mu \mathrm{g}$ of purified thiomuracin GZ was diluted in $20 \%$ aq. MeCN to a final concentration of $37 \mu \mathrm{M}$, and HPLC was performed using a Flexar HPLC system (PerkinElmer) equipped with a BetaBasic C18 column $(4.6 \times 250 \mathrm{~mm} ; 5 \mu \mathrm{m}$ particle size; $100 \AA$ pore size; Thermo Scientific $)$ operating at 1 $\mathrm{mL} / \mathrm{min}$. Following a 10 minute hold at $20 \%$ aq. $\mathrm{MeCN}$, separation was achieved with a gradient of $20-90 \% \mathrm{MeCN}$ over 45 min; purity was assessed by monitoring 220 and $315 \mathrm{~nm}$ (Figure S8).

UPLC-MS and MS/MS of ejected His - $_{6}$-TbtA leader. Purified ejected His ${ }_{6}$-TbtA leader after the macrocylization reaction was dissolved in water, and LC-MS/MS was performed using the same procedure as above. A gradient of $2-100 \%$ aq. $\mathrm{MeCN}$ with $0.1 \%$ formic acid (v/v) over 20 min was used. A ramping of cone voltage of $16-19 \mathrm{kV}$ during the scan was performed to generate peptide fragments for MS/MS analysis.

Kinetic assay to probe if $\mathbf{T b t B} / \mathrm{TbtF}$ accelerate the macrocyclization reaction. HPLC-purified TbtA-hexazoletetradehydrate was dissolved to a concentration of $200 \mu \mathrm{M}$ in $50 \mathrm{mM}$ Tris- $\mathrm{HCl}, 150 \mathrm{mM} \mathrm{KCl}, \mathrm{pH}$ 7.5. Before starting the reaction, enzymes and the substrate were incubated at $30{ }^{\circ} \mathrm{C}$ for $5 \mathrm{~min}$. MBP-TbtF was pre-treated with thrombin in order to release the MBP tag. The reaction was performed with $150 \mu \mathrm{M}$ of substrate 3 and $5 \mu \mathrm{M}$ of either protein at $30{ }^{\circ} \mathrm{C}$. Macrocycle formation was monitored by absorbance at $315 \mathrm{~nm}$. Time points were collected every $2 \mathrm{~s}$ and initial reaction rates were calculated.

Bioinformatic identification of TbtD residues for mutagenesis. The protein sequence for TbtD (gi: 915296818) was used to identify homologs via BLAST searching. Homologs with E-values $<5 \mathrm{E}-50$ were aligned by sequence and highly conserved residues were targeted for alanine replacement.

Enzymatic activity of alanine-substituted TbtD proteins. The activity of the TbtD mutant proteins were assessed under two different reaction conditions with the first using a relatively high concentration of enzyme and substrate and the second using significantly lower concentrations. For the "high" concentration reaction conditions, tandem dehydration $(\mathrm{TbtB} / \mathrm{C})$ and macrocyclization $(\mathrm{MBP}-\mathrm{TbtD})$ reactions were set up identically as previously, with the exception that $100 \mu \mathrm{M}$ of 2 and $5 \mu \mathrm{M}$ of TbtD mutant proteins were employed. For the "lower" concentration conditions, $15 \mu \mathrm{M}$ of 2 and $1 \mu \mathrm{M}$ of TbtD mutant proteins were used. Samples were quenched by the addition of four volumes of MeCN and evaporation to dryness in a Savant SpeedVac system. Samples were then resuspended in a volume of saturated, aqueous guanidine- $\mathrm{HCl}$ equal to the original reaction volume, desalted using $\mathrm{C} 18 \mathrm{ZipTips}$ and eluted in a saturated solution of sinapinic acid in 50\% aq. MeCN. Each reaction was repeated three times, and the relative peak heights of starting material (2) and product (4) were compared by MALDI-TOF-MS.

Enzymatic activity of selected TbtD mutant proteins. Seven TbtD mutants were analyzed in more detail by monitoring the peak area of the EIC (extracted-ion chromatogram) of the macrocycle (ESI, $\mathrm{m} / z$ 1366.2608) produced over time. Experiments with macrocycle standard solutions under the same conditions of the enzymatic activity assay showed that the peak area of the EIC of the macrocycle responded linearly to the macrocycle concentration in a $0.1-25 \mu \mathrm{M}$ range. The following reaction conditions were used for the assay: HEPES pH 7.5 (50 $\mathrm{mM}), 15 \mu \mathrm{M} \mathrm{3}$, and $1 \mu \mathrm{M}$ MBP-TbtD mutant enzyme. The reaction was allowed to proceed at $30{ }^{\circ} \mathrm{C}$, and was quenched by addition of $\mathrm{MeCN}$ to $50 \%(\mathrm{v} / \mathrm{v})$ at different time points. The insoluble material was removed by centrifugation at $16,100 \times \mathrm{g}$ for $5 \mathrm{~min}$, and the supernatant was subjected to LC-MS using a Waters SYNAPT mass spectrometer outfitted with an ACQUITY UPLC, an ACQUITY Bridged Ethyl Hybrid C18 column $(2.1 \times 50 \mathrm{~mm}$, $1.7 \mu \mathrm{m}$ particle size, $200 \AA$; Waters), an ESI ion source, and a quadruple TOF detector. A gradient of 3-100\% aq. $\mathrm{MeCN}$ with $0.1 \%$ formic acid (v/v) over 20 min was used, and the EIC was recorded. 
Table S1: Sequence of $t b t$ biosynthetic genes for optimal E. coli expression. All sequences are provided 5' to $3^{\prime}$. Restriction sites for cloning are underlined (5' BamHI, 3' Xhol). These gene constructs were synthesized by GenScript (Piscataway, NJ, USA).

\title{
tbt $A$
}

GGATCCATGGACCTGAATGATCTGCCGATGGATGTTTTTGAACTGGCAGATAGCGGTGTTGCAGTTGAAAGCCTGACCGCAGGT CATGGTATGACCGAAGTTGGTGCAAGCTGTAATTGCTTTTGTTATATTTGTTGTAGCTGCAGCAGCGCCTAACTCGAG

\begin{abstract}
tbtB
GGATCCATGCGTCTGGTGGAACGTCGCTTTCCGGTTGCCATGACCTCGACGGCTCCGAAAGTCGCAGTGCGTGAATGCGGTCTG CCTGTGAGCGCGATTGAATCTCTGTGCTGTACCGATAGCTTCGCACTGATCCGTCGCCAGGTTCGCGAAACGGCGTGGCTGAAA GGCGAAGGTAAACGTCTGGCCGTGGATCTGGGCCTGCTGATTGGTGAACGTGGTGACGGTGATGATGGTCTGCGTCCGGTTCTG GTCGGTCTGCGTCGCGCACTGCATACCGGTCGTCTGCCGGATGCACGCGAATGGACGCCGCGTGTGGCATCTGCTCTGCCGGCA GAACTGGCAGCCCGCGTCGCTGATTGGGTGACCCGTATGCGTGCACTGACGCGTGCACGTCGCGAACTGCCGGAACTGTTTGCA GCTGAAGCACGCGTTAAAGAAAAAGTCCTGGCACAGGTGGCAGCCGATCCGGGTTTCCGTCGCGCTCTGAGTCTGGCATCCCCG GAACTGGCAGCTGATCTGGACCGTTGGCTGGCAGAACCGGCACGTCGCCCGAAAACCCAAAAACTGCTGCGCCTGGCCAAATAT GTTGCACGTGCGGCCGTCAAAACGTCACCGTACTCGACCTTTACGAGCATGGGTGTGGCCGTTTGGGAAAACGGCGAAGATTGG GCAGACGGTGCTATTGTTCGTTTCGCACCGCGCGAACCGCCGTCAGTCATCCTGGAACCGTCGGGTGAATGGCTGCATGGTGCA CTGCGTGCATGGCTGGCACGTCCGGAAAACCTGGTGCGCAGCCGTCTGCGCCTGAATCCGTCTCTGGTTATTCGCGCTGATAAA GCGGAATTTCTGGGCTTCCCGCCGCGCGAACCGATTATCCGTATGGGTCTGACCCCGGTGGTTGCAACGGTTCTGCGTCTGGCA GAACCGGCAGCTGATGCTGACGGCTGGATCGATCCGATGGGTTTTCGTGATCGTCTGGCTCGTGACCTGCCGGCAGAACCGGAA CAGGTCGACCGTCTGCTGCGCTCCCTGATTGAAGCCGGCGTGCTGGAAGCACACCCGCTGACCCGTGCGGGTCTGCCGGAAACG GGTGAATGGGCAGAAATCCGTGCAGCACTGCGTCATGATCCGCACGGCGAAGACCCGGAAGCGTATCGTGTGCGCCTGGCTCGT CTGAAACGCGCGATGACCATGATGTGGCCGCAAGGTGATACCACGGCGCTGCTGCATGAAACCGCAGTCGTGACGCGTCCGGTT GCAAGCCTGAATCCGACCGCTTGGGGCCGTGGTCTGAGTGATCTGGACGTTGTCCGTCGCTGGCTGTCCGTGTTTGATGGCAAA CTGCCGATTCGCATCGTGGTTGCCGAATATCTGCGTGCACGTTACGGTGAACACGCCCGCGTGCCGTTTCTGACCTTCCATCGT CACGTTCAGGAAGAAATTGCAGGTGATGCACCGTCAGGTGCAGACCTGCGCACGTTCGTTGGTCGTTCGGCAGCTATCTGGGCT CCGCCGCTGGCACATAGCCGCCTGCCGCGTCTGCGTGAACTGGCAAAACTGCGTGAAGCAGCCCGCGAACTGGCACTGGGTCGT CCGGAACACGATGGTATTCAGCGTGTGGACCCGGAAGAACTGATCAAACAAATGGCGACCTGGCCGGAATGGATTGTCGTGCCG CGTTCTTGCGCGTGTTATGTCCAGCCGGCACCGGAAGGCCGCCTGGTGCTGAACGTTGTCCATGGCGGTCACGGTCGTGGTCTG CGTCGCCTGAGTCATCTGATCGGCCGTGTGCGCGGTGAAGCGGTTGATCACCCGATGGTCGCCGACGAACCGGAAGGTACCGTG TACGCGGAACTGAGCGGCTCTCTGGGTTCAACCCTGAATGTCCATGTGCCGGGCACGCGTTATGAAATTGATTACCCGTTTAGT CCGGGTGATCGTTCCCGCGACCGTCGCCTGCCGCTGTCTGACCTGGAAGTGGTTCTGGCACCGGAAACCGGTCTGGCTGAACTG CGCAGTCGTCGCCTGGGCTTTCGTGTTATTCCGCTGCATCTGGGTATGGCAGCTGAATTCCAGCTGCCGCCGGCAGCACGTTTT CTGGAACGTGCCTTCGGCGTGACCTATCTGCTGCACCCGAGTGCACCGCCGCTGCTGCGTATCGGCGAAGTGCCGCCGCCGCAG GAAGTGACGCGTTACCCGCGCGTTGAAGTCGGTCGTGTCGTGGTTCAACGTCGCCGTTGGCTGGCACCGGCAGGTACCCTGCCG ATTCGCGCGAAAGGTGAAGATGACGCCTCCTATCTGCTGCGTCTGGTTGCCTGGACCGATGCAAACGGCATCCCGACGCGTTCA TTTGTTCGCGCCTGGCAGGAACGCATGGTCCAGGCTGGTCAAGATAAAGCGCGTAAACCGCTGTTCCTGGACCTGGCGAATCCG TTTCTGGTGAAAGTTTTCGAACGTCAAATTCGCGATTGTGCATTTGTGCTGTTCGAAGAAGCTCTGCCGGACCCGGCAGACGCT CCGCCGCGCGAAGGCAGCGATCTGCCGCGTGTGATCGAATTTCTGGTTGAACTGGGTGAATAACTCGAG
\end{abstract}

\section{tbtC}

GGATCCATGACCCCGCACGAAGCCGAAGTTATTTGGGTTTCAGCACACATTTTCCATCAAGGCCCGCTGGACCGTCTGGTTACC GAAGTCGCAGGCCCGCTGTTTGTCGAACTGGCAGCAGCAGGTCTGTCCACCCAGGGCTTTTTCCTGCGCTATTGGGAAGGCGGT CCGCATCTGCGTTTCCGTGTTCGTCTGCTGCCGGGTGCAGATGCTGCAGAAACGCGTCGCCTGATTCGCGAACGTGCTTGCGCG TATCTGCGTCGCTACCCGAGCCAGGATGTTCTGGACCGTGAAGAATATGCTCGTCTGGCGGAAGTCCTGGCAGCACGCGAAGGT CTGACCGGTCACGCGCGTGAACTGTACCCGAACGATAGTGTGCAATTTATCTCCTATCGCCCGGAACATCACCGTTACGGTCGT GGTCCGAGCCTGGAAGCAGTTGAACGCCATTTCGCAGAAAGCTCTCGTATTGCTCTGGAACTGCTGCACGCAGGTCTGACCATC CCGCAGCGTGGTGCAGTGTGGCTGACCATGCTGATGGCAACGTGGCTGCTGGCAGGCTCTCGTGGTGAAGATGCGTTTCGCCCG TGGCCGGACGCTCGTAAAGAACAAGCGTTCGATCGTGAACGTGAACGTCTGACCGGTCTGGCACGTCATGCACGTCAGCTGACG CTGCGCCCGCCGGACCCGGAAGCACAAGGCGTCCTGGCTGCATGGCACGGTTCAGTGGCACGTCTGGCAGATGCACTGGCAGCA TCGGGCTTTACCGCCACGCGTACCGCAACGGTTCTGGACCTGTGTGCTCATCTGCTGGCGAATCGTCTGGGTATTCGCATCCAG GATGAAGCACGTCTGCGCTACCTGGCTAGTCGTGCGCTGGACGAAGCGGAAGTGACCGTTTAACTCGAG

\section{tbtD}

GGATCCATGGCAGCAGGTGAACGTTGGTGGCGTTTCCGTGTCGATTATCATGCAGGCCCGATGGACGATCTGATTCTGGATGGT GTGCGCCCGGCATTTGCAGCATTCGCAGCTCAGGCACCGATGGCATATTTTCTGCGTCATTGGCGTCGCGGTCCGCACCTGCGC ATTTACGTTAGTACCACGCGTGAAGCACTGGAAGCTGTGGTTCGTCCGGCAATCGAACATGTCGTGGGCGGTTATCTGCGTGCA CGTCCGAGTCCGGGTATGGCTGATCCGTCCGCGTTCCTGCCGCTGCATGAACGCCTGGCCGAACTGGAAGGCGAAGATGGTCCG CTGATGCCGTGGTCTCCGGACAACACGATTCACGCAGAAGGTGAACGTCCGGAACCGCTGACCGTGCGTGATGTTCTGCTGGCC GATTTTTATGCAGACACCACGCCGAGCGTTTACCACGCGCTGGAACGTGTCCGCAGTGGTGCTTCCCTGCCGACGATCGCCTTC GATCTGGTTGTCGCTACGGCACATGCACTGAGTACCGGCGGTCTGCCGGTCGCACGTACCTCACTGCGTTCGCACGCAGAAGCT TATCTGGCACGTCGCTCCGATGGTGTGCGTCTGCGTGAACTGTGGCGTGACCATTACGCCCGTAACCGCGAAGCATTTACGGAA CGTCTGATTGCGGTGGCCAGCTCTGCGGAAAGCGCCGAAAATGGTGCGCATCTGCCGCACGTCCGTGAATGGGTGCGTCGCCTG CGTCCGATCCGTGAACGTGCACGTGCTCTGCTGGAATCAGGCGAACTGACCCTGGAATATGCATCGCCGGCTGAAGGTGCGCGC 
GATCTGCCGAGCCTGGCTGAAGTTTCTGCGTTTCATCGCGAACTGGAAAGCCGTCCGGAATGGGCACGTCTGCGTGACTCTCCG GCATTCGGCGCATATCGTCTGGTTATTAATTGCACCTACCTGCACCTGACGCGTCTGGGTCTGACCCCGCATCAACGTTTCCTG GTCTGTCACCTGGCAGCAGATGCAGCTGCAGACGTGTACGGCATCGCCGCACATGAAGAAGTTGCGACCCGTTAACTCGAG

\section{tbtE}

GGATCCATGACCATTCCGCCGGGCCTGACCGAACGCTATGCCCTGCGTGCGGGTGTTCACTCTGCTGTGCTGCCGGACGGTGTT ATGCGCCTGTTTGCGTGGCCGCATGCCGAATCAATTGGTGCGCTGTCGGCCGATGAAACCACGCTGCTGAAAGAACTGGCGGAA GGCCCGCGCGAAATCGCAGATCCGGCTCTGCGTCCGTTTGTGGAACGCCTGTTCCGTGGCGGTTGGCTGAAACGCACCCTGAGC CGTGGCGAACACGACCTGTATACGCTGGACCCGCTGCGTCGCCCGGGTAGTCGCCCGGCACCGCCGGATGACCCGGTCCTGTCC CGTTTTGCAGCAGTGCGTCGCCGTCCGAGCGGTTTCGTTATTGAATCTCCGCTGGCGTGGTGCGACGTGCATGTTCACGATCCG GCACTGCTGCCGGATCTGCTGGAACCGGCAGGCGGTCGTGCTGGTCGTAGCTCTCTGGCACCGCAGATCCGCCGTCAAGCACTG GCTGATCTGGCATGGGCAGGCCTGGTGGTTCCGCGTGGCGCAGAAGACGGTGCACTGCGTACCCGTCAGTGGGCACCGCATGAA CTGGATTTTCACCAACGCTCCCGTCTGTATCATCGCGGCTACCTGGGCGATGGTTTTGGCGGTACCTTCTGGGCACGTGGTACC TTCGACCCGCCGCAGGCTCGTCCGCAACGTTATCCGGGCGACCCGATTCCGCTGCACCGTCCGGATCTGAACGCACTGCGTGCA GCTGACCCGCCGCTGACCGCTGTTCTGGAAGATCGCCGTAGCGTCCGTGAATATGATGACGATGCACCGATGACGGTGGAACAG CTGGGTGAACTGCTGTACCGCTCTGCGCGCATTCGTGACGTCAAAGTGATCGATGGCGTGGAATATGTTCGTAAACCGTACCCG TCAGGCGGTTCGGTGTATGAACTGGAAATTTACCCGGTCGTGCGTCATGTTGCAGGTCTGGCACCGGGCATGTATCATTACGAT GCGTATGAACACGTTCTGCGTCCGGTCCGTCCGGCAGGTCATCCGGCTGTGCGCCGTATGCTGACCGTTGCAAGCCACGGCTCT GCAGTGGGTATCCGTCCGCAGCTGCTGCTGGTTGTCAGTGCCCGCGTCGGTCGTGTGATGTGGAAATATGAAGGCATGGGTTAC GCACTGATTCTGAAACATGTTGGTGTCCTGTATCAAACCCTGTACTGCGTTGCAACGGCTATGGGCCTGGCACCGTGTGCTATC GGCAGTGGTGATTCCGCGGCCTTTTCTGAAGCGACCGGTCGCGATCCGCTGGAAGAATGTGCCGTCGGCGACTTCCTGCTGGGT TCACGTCCGGCGAGTGGCTCCACGGTTGAAGAATCGTAACTCGAG

\section{tbtF}

GGATCCGTTATTCCGGCTCGTCCGGTTCTGACCCCGGGCGTTCGTTATGCTCCGACCAGTGATGGCGTTGCTTTCCTGACCCGT GATGGTATTGTGAGTATTACCGGCGTTTCCATCCACCGCTGGATTGACCGTATCGCACCGCATCTGACCGGTGAACGCAGCGTT GCAGAACTGACGGATGGTCTGGCACCGGAACGTCGCGCATTCGTGCTGCGTCTGCTGCAGGCACTGGCAGATCGCGGTCTGATT ATCGACGCAGCCGGCGGTCGTGAACCGCGTCTGGAACGTGGTACCGCTTGCGCACTGCACGTTACCGAAACGTGTGGTCCGTAT CGTGAATCACTGCCGAAACTGGCACGTTCGCTGGCAGATGCACTGACCCGTGCCGGTCTGGACGTGCGTCTGGCTAGTGCGTCC GAACCGTGCGATCCGGAACGTTGTCATGCGCTGGTTCACCTGACGGCAGCTGACGAAGTCGGTCTGGCAGCAGCAGAACGTCTG GATCGTCTGTCAGAACGTTGGGGTGTTCCGATTGCACATGTGGTTGTCTGGGGCGGTGAAATCTGGCGTACCGAAGCCGGTACG GTGGGTGAAGACGGTCTGCCGCTGGCTGCAGGTTGGCGTCGCCTGACCGCGCTGCGTCCGGATACGGAAGAACGCGTTCCGATT TCGCCGACCGCAGCAGTGGTTGTCGCCGGTCAGGTCGCTGCAGATGTGCGTGACCGTCTGACCACGGCAACCCGTGCTGGTCGT CGCCCGCGTCTGCACGTGGTTGAACCGGCTCGTCTGACGTGCCGTGCACATCACTTTATTCCGCATTTCCACACCATGCCGCTG ACGGGTGATGCACCGCTGGCAGAAGAACTGACCGAAGAAGAATTTTCCCGTCGCGCCGCAGCTCTGATGGATGACCGCACCGGT GTGTTTACGGAAATCGCGGAGGGTGATTTCGGCCAACTGCCGCTGCATGTGGCTGTTACCACGGTTGCCGATCCGGTCGGTCTG CTGGGTCCGGCAGGTGACCGTCCGCGCGTGATTGGCGTTGGTCCGGATTTCGCAACCGCACGTTATCGTACGGCGCGTGCGGCA ATCGCACTGTACGGTCTGCTGGCAGTTGATCCGCGTCGCCTGGTCGATGCAGACGGTGAACCGCTGGCAGGTCCGCGTACCAGC GCAGCTGAACTGGAAGATCTGCTGGGTCGTATTCGCGCGGGCGGTCTGCCGGCATTTGTCCGTGCCGAAGATGTGCGTGGCGGT CCGCCGCGTCTGCTGCCGGCCAGCTCTGTGTTTCCGTTCGCTACCGCGCGTGCCCTGCTGGCCGGTACGGTGCCGACCGGCACG GCAGTTGGTTACTCTGAAGAAGGTGCACTGCGTGCAGCACTGCTGGATCACTGCCGTGAACGCACCCTGGCAGACCTGGCTAGT CGTCGCGTCGCTCGTCTGACCCCGGATACGGCACCGTCCGACCCGGTGACCGCACGTTATCTGGCTTTTCTGCGCGCGATCGGC CTGCCGTTCGATGTTCTGGACGCAACGGGTCCGCTGGGTGTCCCGGTGTACGTTGGCGTCCTGGATGGTCGCGTCGTGGCAGCT GAAGCAGGTGCTAGCCCGGGTGAAGCGTTTCGTTCTGTGCTGGAAGGTATTCTGCGCGGCCTGCAAGGTATCCCGGCAGCACGT AGTCTGCCGGACACCCTGCCGCCGGTCCTGCCGGAAGCAATGGCAGTGCCGGATGGCAGCCCGCTGTCTGCAGAAGCTCTGGCA GCTGCACTGGCGCGTGCCGGTCTGTCAGTGTCGGTTGTCCGCCTGGATCATGACCGTCGCGTTCACGCCCTGATTCCGCATTGT GTGCGTGTTATGGTCACCGGCGAAGAATCTGCACATGTGTAACTCGAG

\section{tbtG}

GGATCCATGTCCGACGCTCTGGCTGCTGCTATTATTGGTGCTGGTCTGCTGGCCGATGAACTGGCTGCTGCCTTTCGCCCGTAT GGTGAAGTCATTCGTCTGGATGAAGTCGAACGCGCTGAAACCGAAGCGCATCGTCTGCGCGCCCTGGTTATGGCATGCGATGGC TGGGACACCAGTATTTACCGTGAAACGCGTGAACGCTGCCGTCGCCTGGGTATCCCGTGGCTGCCGGTTCGTACGGAACATGGC ATTGCAGTCATCGGTCCGCTGGAACGTCCGGGTGAACCGGGTTGCGCCCACTGTTTTGAACTGCGTCGCGAACGTGCACGTCCG GATGCAGCCGCATACCGTGCAATCCTGAACCATCACGGTCCGGAACTGGCAAAACGTCCGTCACCGTATCTGGACGAACTGGCT GCGGCCACCATTGCAGCTCTGGGTGTGCGTATGATCGATCAGGGCTCGGGTTGCCATGTCTGGTATGTGCGTCTGAATGGCCTG ACGGTTGAACGTCACGCGTTCCTGCCGGAACCGCTGTGCCCGGAATGTGGTAGCCTGCCGATGGATGACCGCGATAGCGCTGTT ATTGTCCTGCGTTCTCAGCCGAAACGCGCCCCGGATGACTACCGTACCCGCGATATCGTGGACGAACTGGATGCCCTGGTTGCA GACTATGTCGATGGCGAATCTGGTCTGATTCGCCCGCTGGTGCGTGATACCCAAGGCGGTCTGGTTATCGCAGGTGCAATGCTG CCGCTGCGTTTTGCGGGCGGTTCAGAACCGGGCGTTGGTCGTACGCGCGGTTATCGTACCTCGGAAGTCACGGCTATTCTGGAA GCGCTGGAACGTTGGGGCGGTGTCGAACCGGGCGGTAAACGCACCGTGATTCGTGCGGCCTTCCGCGATATCGCAGCTGACGCA CTGGACCCGCGTACGCTGGGCGTGCATAGCCCGGAAGCATACGCGGCCGAAGACTTTCCGTTCCGCCCGTTTACCGAAGATGAA GAAACGGAATGGGTGTGGGGTTATAGTTTCGCACGTCGCTCCCCGATTCTGGTGCCGGAAACCGTTGCGTATTACTATGTTCAC CGTGACCGCCCGCGTCAAGAACGTCCGTTTCTGTATGAAATCAGTAACGGCTGCGCTCTGGGCTCCGGTATGGAAGAAGCGATT CTGTACGGTATCCTGGAAGTGGTTGAACGCGATGCGTTCCTGATGACCTGGTATGGCCGTCTGGGTGTTCCGCGCATTGATCTG GACAGCGCAAAAAATCGTATGGTCCCGCTGCAGGCAGCTGCGATTTCTGCGGAAACCGGCTACCGCATCGAAGTCTATGATACC ACGATGGAACATGGTATTCCGAGCGTGTGGGCTCTGGCAATCCGTGGCGGTGAAGAACCGACGGCAGCACCGCGTCCGCGTATG 
GTGTGTGCTGCAGCAGCACACCTGGACCCGGAACAAGCAGTTCTGAGCGCACTGTCTGAACTGGGTCCGCTGCTGGCAGACCTG CTGCGTCGCTACCCGGATGAAGCTGAACGTGCGCGTCGCATGGTCGATGACCCGGGTCTGGTGACCACGATGCATGACCACAGT ACCCTGTATGGCGCCGATGAAGCATTTGGTCGCCTGGAATTCCTGACCGGTACGGCATCCACCCGTGACCTGGCTGCAATGCGC GAATCAACGGTGGCTTTTCGTTCGGCGGATCTGCGCGATGACCTGCTGGAAGTGATTGGCCGTATCCTGGCGGAGGGTATGGAC GTTATTGTCGTGGATCAGACCACGCCGGAACATCGCGTGCACGGTTTTACCTGTGTGAAAGTTCTGATTCCGGGCATGATCCCG ATGACCTTCGGTCATCGTAACCGTCGCCTGGATGGTCTGACGCGTCCGCTGGTTATCCCGTACCGTCTGGGTTATCGTCCGGCA CCGCTGACCCCGGAAGCACTGAATCCGCATCCGCACCCGTTTCCGTAACTCGAG 
Table S2: Oligonucleotide primers used in this study. All sequences are provided 5' to 3'. F indicates a forward primer while $\mathrm{R}$ indicates the reverse. Lowercase $\mathrm{m}$ indicates $2^{\prime} O$-methylation of the following residue; methylation suppresses random addition of bases at the end of the RNA by T7 RNA polymerase. ${ }^{1}$

\begin{tabular}{|c|c|}
\hline Primer Name & Oligonucleotide Sequence \\
\hline$m b p \mathrm{~F}$ (sequencing) & GAGGAAGAGTTGGCGAAAGATCCACGTA \\
\hline T7 F (sequencing) & TAATACGACTCACTATAGGG \\
\hline T7 R (sequencing) & GCTAGTTATTGCTCAGCGG \\
\hline tbtA Gibson $\mathrm{F}$ & GCATCACCATCATCACCACAGCCAGATGGACCTGAATGATCTGCC \\
\hline tbtA Gibson $\mathrm{R}$ & GACTTAAGCATTATGCGGCCGCATTAGGCGCTGCTGCAG \\
\hline tbtB Gibson F & CTGGTGGACAGCAAATGGGTCGCGGAATGCGTCTGGTGGAAC \\
\hline tbtB Gibson R & GTGGTGGTGCTCGAGTGCGGCCGCATTATTCACCCAGTTCAACCAG \\
\hline tbtC Gibson F & CTGGTGGACAGCAAATGGGTCGCGGAATGACCCCGCACGAAG \\
\hline tbtC Gibson R & GTGGTGGTGCTCGAGTGCGGCCGCATTAAACGGTCACTTCCGCTTC \\
\hline E. coli gluRS Gibson $\mathrm{F}$ & CTGGTGGACAGCAAATGGGTCGCGGAATGAAAATCAAAACTCGCTTC \\
\hline E. coli gluRS Gibson $\mathrm{R}$ & GTGGTGGTGCTCGAGTGCGGCCGCTTACTGCTGATTTTCGCG \\
\hline E. coli tRNA F & AATTCCTGCAGTAATACGACTCACTATAGTCCCCTTCGTCTAGAGGCCCAGGACACC \\
\hline E. coli tRNA R & mUmGGCGTCCCCTAGGGGATTCGAACCCCTGTTACCGCCGTGAAAGGGCGGTGTCCTGG \\
\hline T. bispora tRNA(CUC) F & АATTCCTGCAGTAATACGACTCACTATAGGTCCCGTCGTCTAGAGGCCTAGGACGC \\
\hline T. bispora tRNA(CUC) R & mUmGGTAGTCCCGAGCGGATTCGAACCGCCGTTACCGCCTTGAGAGGGCGGCGTCCTAGG \\
\hline T. bispora tRNA(UUC) F & AATTCCTGCAGTAATACGACTCACTATAGCCCCCATCGTCTAGCGGTCTAGGACACCGCC \\
\hline T. bispora tRNA(UUC) R & mUmGGTGCCCCCAACGGGATTCGAACCCGTGCCGCCGCCTTGAAAGGGCGGTGTCC \\
\hline tbtG Internal & GTGATACCCAAGGCGGTCT \\
\hline tbtF Internal & TGCACATCACTTTATTCCGCA \\
\hline tbtB Internal 702 & CTTTACGAGCATGGGTGTGG \\
\hline tbtB Internal 1496 & TCCATCGTCACGTTCAGGAA \\
\hline tbtB Internal 2116 & GTTATTCCGCTGCATCTGG \\
\hline pbtD (BamHI) F & AAGGATCCGTGACCTGGCGACGCTTTGAC \\
\hline pbtD (Xhol) R & AACTCGAGTCACATGCCTCCTACGGCGATC \\
\hline$t b t D \mathrm{H} 46 \mathrm{~A} \mathrm{~F}$ & АTTTTCTGCGTGCTTGGCGTCGCGGTCCGCACCTGCGCATTTAC \\
\hline tbtD H46A R & CCGCGACGCCAAGCACGCAGAAAATATGCCATCGGTGCCTG \\
\hline tbtD W47A F & TTCTGCGTCATGCGCGTCGCGGTCCGCACCTGCGCATTTAC \\
\hline tbtD W47A R & GGACCGCGACGCGCATGACGCAGAAAATATGCCATCGGTGC \\
\hline$t b t D$ R49A F & GTCATTGGCGTGCCGGTCCGCACCTGCGCATTTACGTTAG \\
\hline$t b t D \mathrm{R} 49 \mathrm{~A} \mathrm{R}$ & CAGGTGCGGACCGGCACGCCAATGACGCAGAAAATATGCCATC \\
\hline$t b t D \mathrm{H} 52 \mathrm{~A} \mathrm{~F}$ & GTCGCGGTCCGGCCCTGCGCATTTACGTTAGTACCACGCGTG \\
\hline tbtD H52A R & GTAAATGCGCAGGGCCGGACCGCGACGCCAATGACGCAG \\
\hline$t b t D$ R54A F & GTCCGCACCTGGCCATTTACGTTAGTACCACGCGTGAAGCAC \\
\hline tbtD R54A R & CTAACGTAAATGGCCAGGTGCGGACCGCGACGCCAATGACG \\
\hline$t b t D$ S85A F & GTGCACGTCCGGCTCCGGGTATGGCTGATCCGTCCGCGTTC \\
\hline$t b t D$ S85A R & GCCATACCCGGAGCCGGACGTGCACGCAGATAACCGCCCAC \\
\hline$t b t D \mathrm{H} 98 \mathrm{~A} \mathrm{~F}$ & GTTCCTGCCGCTGGCTGAACGCCTGGCCGAACTGGAAGGCGAAG \\
\hline
\end{tabular}




\begin{tabular}{|c|c|}
\hline$t b t D$ H98A R & GCCAGGCGTTCAGCCAGCGGCAGGAACGCGGACGGATCAGC \\
\hline$t b t D \mathrm{E} 105 \mathrm{~A} \mathrm{~F}$ & CTGGCCGAACTGGCAGGCGAAGATGGTCCGCTGATGCCGTGG \\
\hline$t b t D$ E105A R & ССATCTTCGCCTGCCAGTTCGGCCAGGCGTTCATGCAGCGG \\
\hline tbtD N118A F & GGTCTCCGGACGCCACGATTCACGCAGAAGGTGAACGTCCGG \\
\hline$t b t D$ N118A R & GCGTGAATCGTGGCGTCCGGAGACCACGGCATCAGCGGACC \\
\hline$t b t D \mathrm{~T} 144 \mathrm{~A} \mathrm{~F}$ & TTTATGCAGACGCCACGCCGAGCGTTTACCACGCGCTGGAAC \\
\hline$t b t D \mathrm{~T} 144 \mathrm{~A} \mathrm{R}$ & ACGCTCGGCGTGGCGTCTGCATAAAAATCGGCCAGCAGAAC \\
\hline tbtD R189A F & GTACCTCACTGGCTTCGCACGCAGAAGCTTATCTGGCACGTC \\
\hline tbtD R189A R & TCTGCGTGCGAAGCCAGTGAGGTACGTGCGACCGGCAGACC \\
\hline tbtD S190A F & СCTCACTGCGTGCGCACGCAGAAGCTTATCTGGCACGTCGC \\
\hline$t b t D \mathrm{~S} 190 \mathrm{~A} \mathrm{R}$ & GCTTCTGCGTGCGCACGCAGTGAGGTACGTGCGACCGGCAG \\
\hline tbtD H191A F & CACTGCGTTCGGCCGCAGAAGCTTATCTGGCACGTCGCTCC \\
\hline$t b t D \mathrm{H} 191 \mathrm{~A} \mathrm{R}$ & TAAGCTTCTGCGGCCGAACGCAGTGAGGTACGTGCGACCGG \\
\hline$t b t D$ E193A F & GTTCGCACGCAGCAGCTTATCTGGCACGTCGCTCCGATGGTG \\
\hline$t b t D$ E193A R & GCCAGATAAGCTGCTGCGTGCGAACGCAGTGAGGTACGTGC \\
\hline tbtD S287A F & TGGCTGAAGTTGCTGCGTTTCATCGCGAACTGGAAAGCCGTC \\
\hline$t b t D$ S287A R & CGATGAAACGCAGCAACTTCAGCCAGGCTCGGCAGATCGCG \\
\hline tbtD H290A F & GTTTCTGCGTTTGCTCGCGAACTGGAAAGCCGTCCGGAATGG \\
\hline$t b t D \mathrm{H} 290 \mathrm{~A} \mathrm{R}$ & TCCAGTTCGCGAGCAAACGCAGAAACTTCAGCCAGGCTCGG \\
\hline$t b t D$ R291A F & CTGCGTTTCATGCCGAACTGGAAAGCCGTCCGGAATGGGCAC \\
\hline tbtD R291A R & СTTTCCAGTTCGGCATGAAACGCAGAAACTTCAGCCAGGCTC \\
\hline$t b t D$ N316A F & GTCTGGTTATTGCTTGCACCTACCTGCACCTGACGCGTCTG \\
\hline$t b t D \mathrm{~N} 316 \mathrm{~A} \mathrm{R}$ & CAGGTAGGTGCAAGCAATAACCAGACGATATGCGCCGAATGC \\
\hline tbtD Y319A F & TTAATTGCACCGCCCTGCACCTGACGCGTCTGGGTCTGACC \\
\hline$t b t D$ Y319A R & GTCAGGTGCAGGGCGGTGCAATTAATAACCAGACGATATGC \\
\hline$t b t D \mathrm{R} 324 \mathrm{~A} \mathrm{~F}$ & CTGCACCTGACGGCTCTGGGTCTGACCCCGCATCAACGTTTC \\
\hline$t b t D \mathrm{R} 324 \mathrm{~A} \mathrm{R}$ & GTCAGACCCAGAGCCGTCAGGTGCAGGTAGGTGCAATTAATAAC \\
\hline tbtD R332A F & CCCCGCATCAAGCTTTCCTGGTCTGTCACCTGGCAGCAGATG \\
\hline$t b t D$ R332A R & CAGACCAGGAAAGCTTGATGCGGGGTCAGACCCAGACGCGTC \\
\hline tbtD C336A F & GTTTCCTGGTCGCTCACCTGGCAGCAGATGCAGCTGCAGAC \\
\hline$t b t D$ C336A R & GCTGCCAGGTGAGCGACCAGGAAACGTTGATGCGGGGTCAG \\
\hline tbtD H337A F & TCCTGGTCTGTGCCCTGGCAGCAGATGCAGCTGCAGACGTG \\
\hline$t b t D \mathrm{H} 337 \mathrm{~A} \mathrm{R}$ & TCTGCTGCCAGGGCACAGACCAGGAAACGTTGATGCGGGGTC \\
\hline
\end{tabular}


Figure S1: SDS-PAGE analysis of proteins used in this study. The minor impurity found at $\sim 45 \mathrm{kDa}$ is the result of endogenous protease activity on the MBP fusion proteins. Cleavage occurs in the linker region between MBP and the protein of interest. Enrichment occurs due to the use of amylose resin for affinity purification.
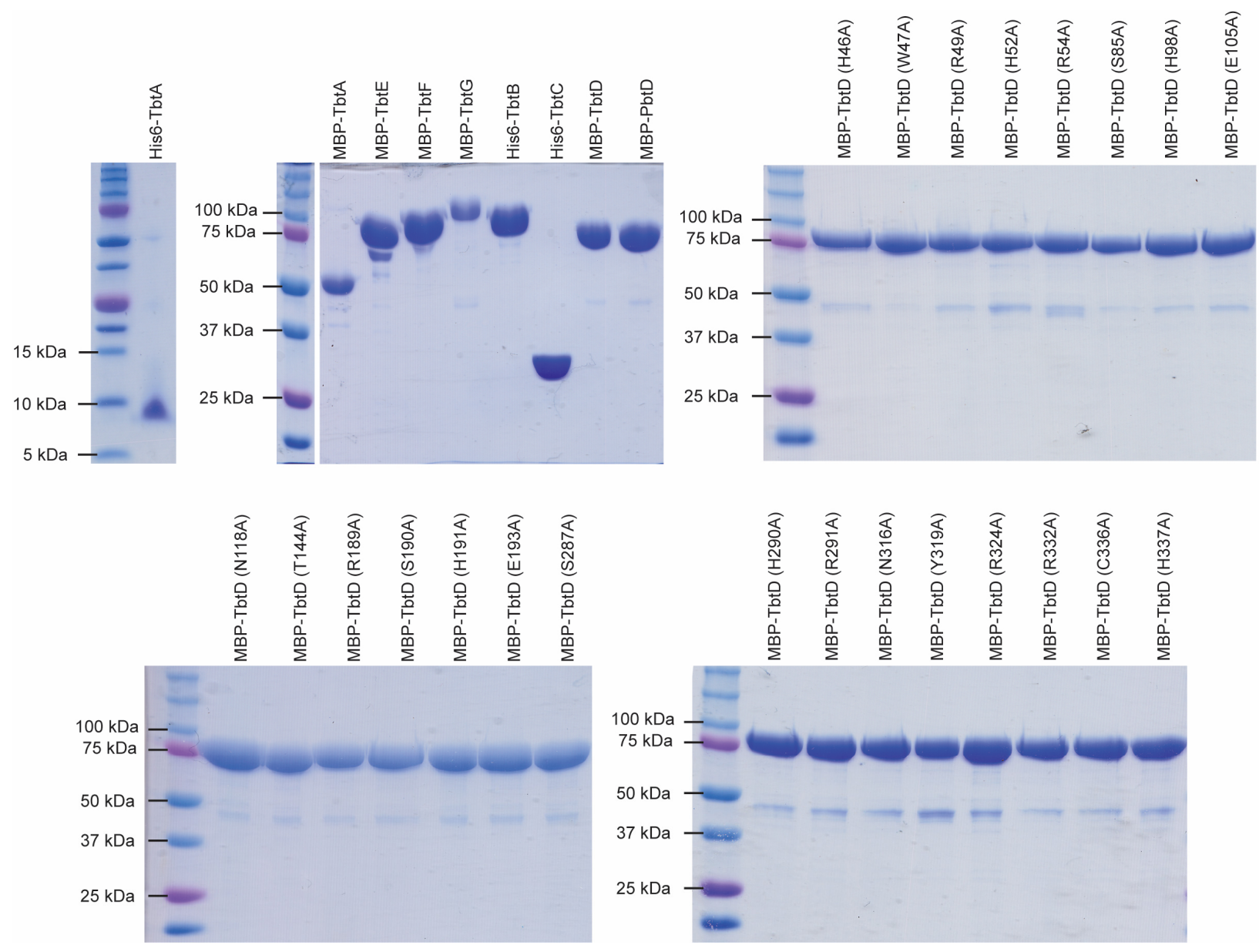
Figure S2: TbtB/C do not process unmodified TbtA. The top MALDI-TOF mass spectrum shows unmodified TbtA precursor peptide (1). The middle spectrum spectrum shows 1 treated with $\mathrm{TbtB} / \mathrm{C}$ in the presence of other necessary reaction components $\left[E\right.$. coli GluRS and $E$. coli $\mathrm{tRNA}^{\mathrm{Glu}}$. The bottom spectrum is an equivalent reaction except with $T$. bispora $\mathrm{tRNA}{ }^{\text {Glu }}(\mathrm{CUC})$. These data suggest that the split LanB dehydratase (TbtB/C) requires a thiazol(in)econtaining substrate, implicating cyclodehydration $(\mathrm{TbtF} / \mathrm{G})$ and dehydrogenation $(\mathrm{TbtE})$ as the initial two steps of thiopeptide biosynthesis.

His6-TbtA: PHHHHHHSQVDLNDLPMDVFELADSGVAVESLTAGHGMTEVGA*SCNCFCYICCSCSSA

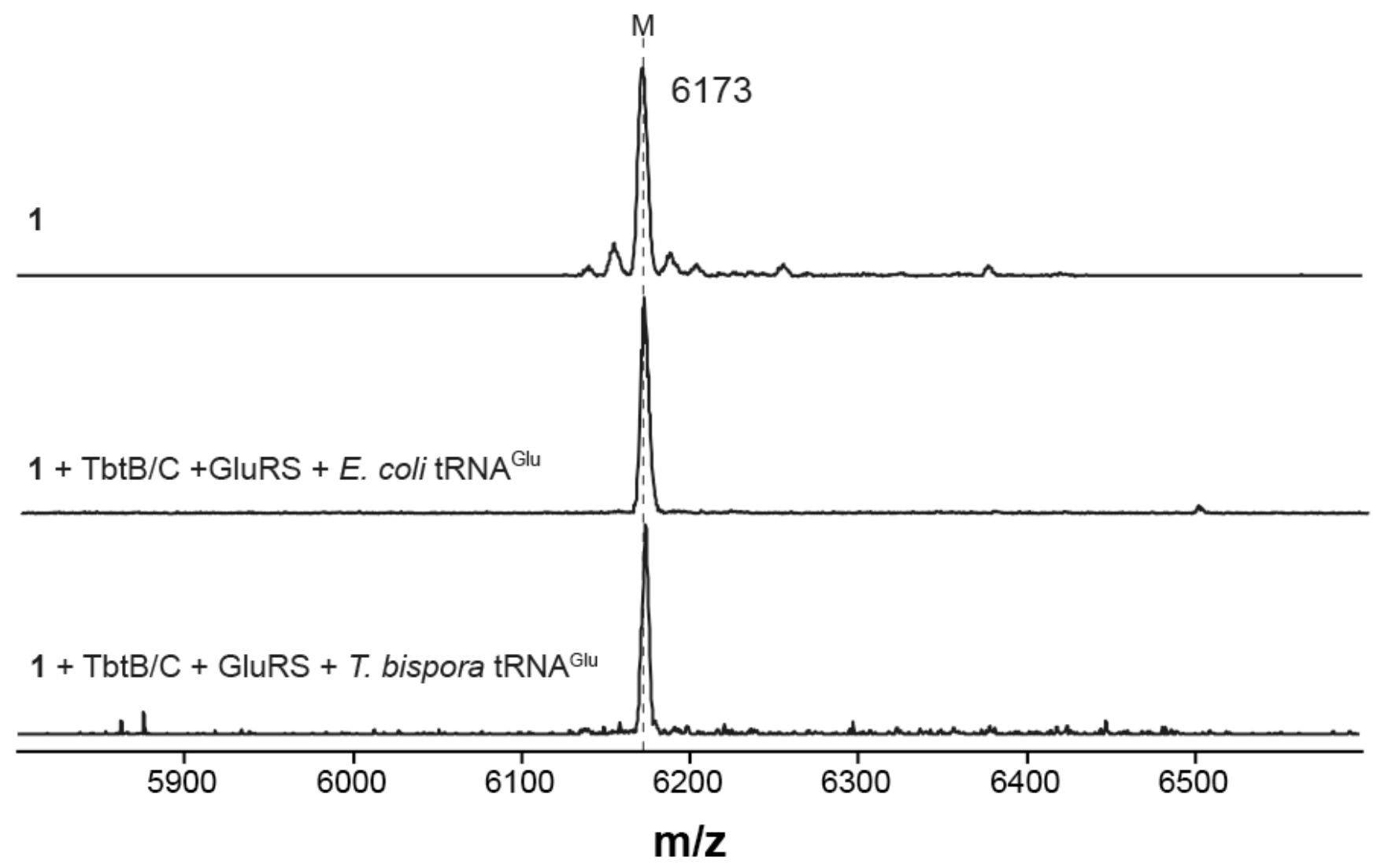


Figure S3: MALDI-TOF-MS analysis of TbtE/F/G reaction requirements. Unmodified TbtA (1) is shown in the top spectrum. Reaction of $\mathbf{1}$ with the F-protein dependent cyclodehydratase (TbtF/G) and ATP results in a six-fold cyclodehydrated product (hexa-thiazoline). Addition of the dehydrogenase ( $\mathrm{TbtE}$ ) to an otherwise identical reaction yields a 6-fold dehydrated and 6-fold dehydrogenated product (hexazole, 2). Omission of ATP, a requirement of the cyclodehydratase, results in no product formation. Similarly, omission of either TbtF or TbtG results in no product formation. Residues undergoing thiazol(in)e formation in the complete reaction are colored blue in the sequence above the spectra.

His6-TbtA: PHHHHHHSQVDLNDLPMDVFELADSGVAVESLTAGHGMTEVGA*SCNCFCYICCSCSSA

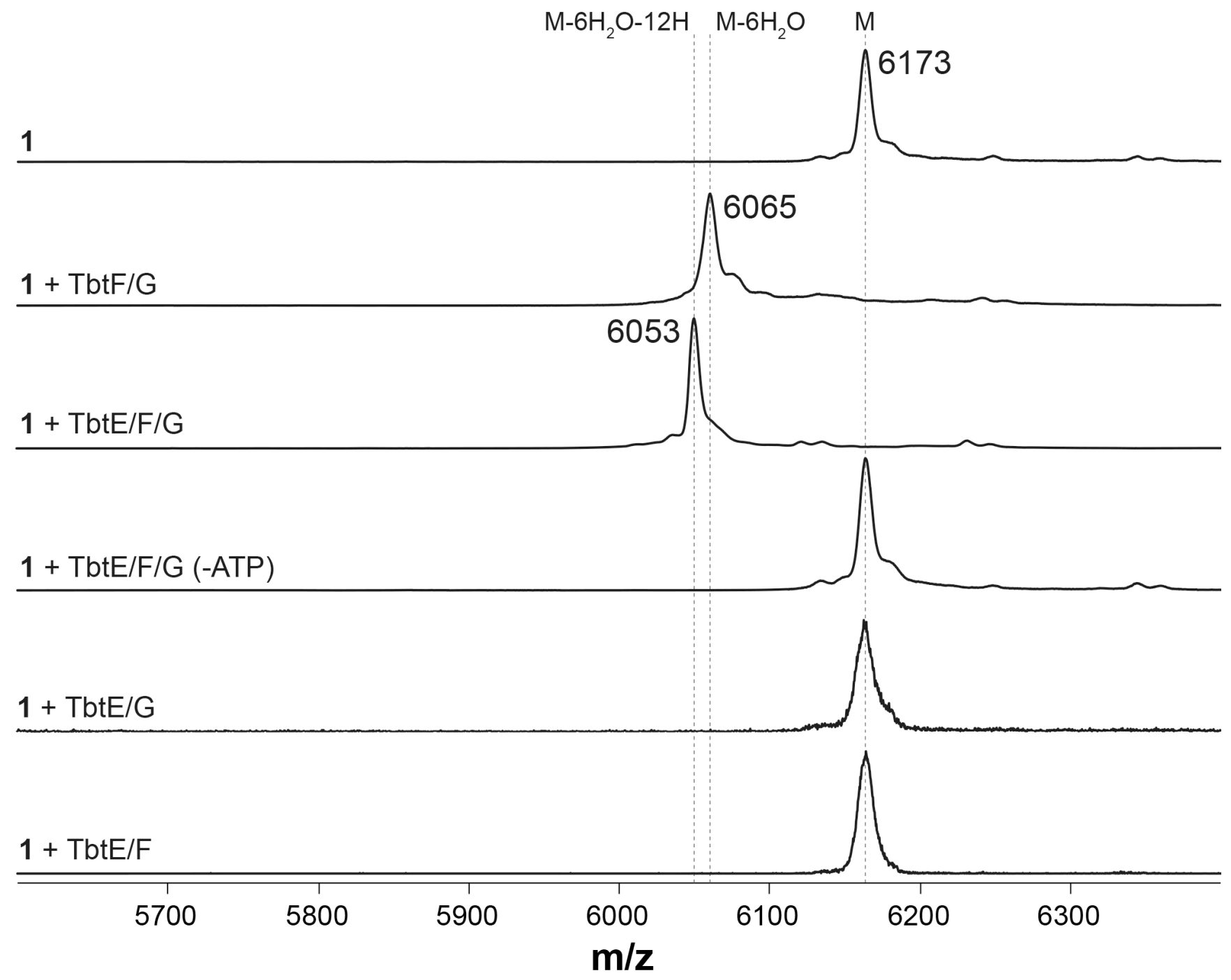


Figure S4: $\mathrm{TbtB} / \mathrm{C}$ is selective for a particular tRNA ${ }^{\mathrm{Glu}}$. (A) MALDI-TOF mass spectrum of 2 (starting material). (B) E. coli $\mathrm{tRNA}^{\mathrm{Glu}}$ (UUC) is not accepted by $\mathrm{TbtB} / \mathrm{C}$. (C) In contrast, four dehydrations are observed upon treating 2 with $\mathrm{TbtB} / \mathrm{C}$ and $\mathrm{tRNA}{ }^{\mathrm{Glu}}(\mathrm{CUC})$ from $T$. bispora. (D) An identical reaction with the alternative tRNA ${ }^{\text {Glu }}(\mathrm{UUC})$ from T. bispora yielded a mixture of dehydrated species, primarily the didehydrated peptide. (E) A reaction that omitted TbtC (elimination domain), while employing the optimal tRNA and $d_{5}$-Glu, yielded a mono-glutamylated species. (F) Sequence alignment of the three pertinent tRNA ${ }^{\text {Glu }}$. Top, T. bispora tRNA ${ }^{\text {Glu }}$ (CUC); middle, T. bispora tRNA ${ }^{\text {Glu }}(\mathrm{UUC})$; bottom, E. coli $\mathrm{tRNA}^{\mathrm{Glu}}$.

His6-TbtA: PHHHHHHSQVDLNDLPMDVFELADSGVAVESLTAGHGMTEVGA*SCNCFCYICCSCSSA

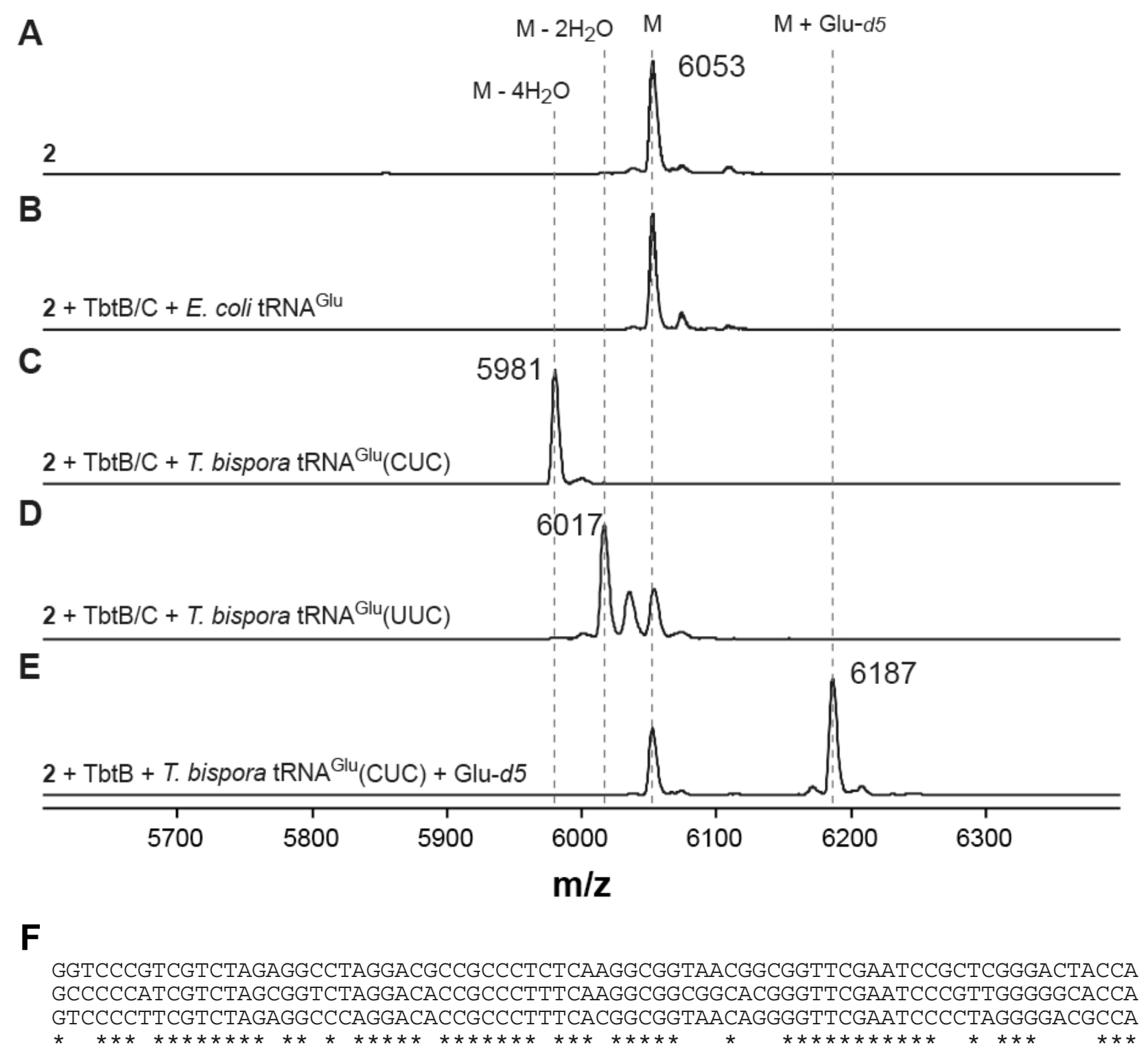


Figure S5: MALDI-TOF-MS analysis of TbtB/C reaction requirements. Shown in the top spectrum is hexazole 2, which is the six-fold cyclodehydration and dehydrogenation product of TbtA. Reaction of 2 with the split LanB dehydratase $(\mathrm{TbtB} / \mathrm{C})$ in the presence of $E$. coli GluRS and T. bispora tRNA ${ }^{\mathrm{Glu}}(\mathrm{CUC})$ results in a four-fold dehydrated product (3). The formation of $\mathbf{3}$ was dependent on the presence of all reaction components, as individual omission of TbtB/C, E. coli GluRS, or T. bispora tRNA ${ }^{\text {Glu }}(\mathrm{CUC})$ did not result in any change in mass. Residues modified to a thiazole are shown in blue, and residues that are transformed to dehydroalanine are colored green. All reactions also contained Glu, ATP, and $\mathrm{MgCl}_{2}$ (see methods).

His6-TbtA: PHHHHHHSQVDLNDLPMDVFELADSGVAVESLTAGHGMTEVGA*SCNCFCYICCSCSSA

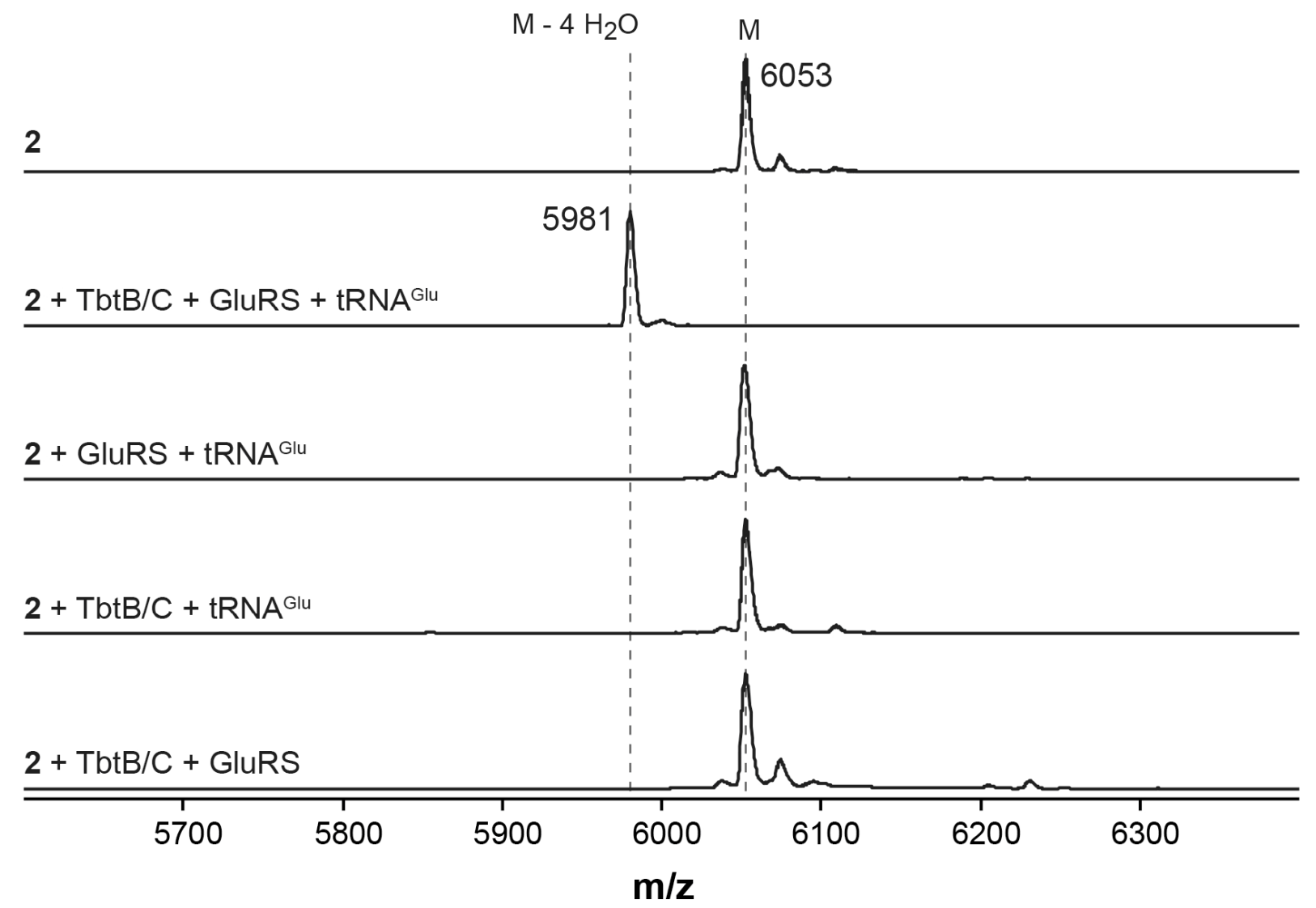


Figure S6: TbtD requires the leader peptide for activity. (A) HR-ESI-MS of the TbtA leader peptide generated by macrocyclization (theoretical $[\mathrm{M}+\mathrm{H}]^{+}, 4593.143 \mathrm{Da}, 0.4 \mathrm{ppm}$ ). The sequence of the His ${ }_{6}$-TbtA-derived substrate 3 is given above panel A with modifications present color-coded (blue, thiazoles; green, dehydroalanines). The asterisk designates the end of the leader peptide. Shown italicized are additional N-terminal residues present on the substrate (His-tag and cloning remnants). (B) MALDI-TOF-MS of 3 after treatment with endoproteinase GluC. This treatment removes all but three residues (VGA, boldfaced and underlined) of the leader peptide. (C) Reaction of GluC-treated 3 with TbtD. No reaction was observed.

His6-TbtA: PHHHHHHSQVDLNDLPMDVFELADSGVAVESLTAGHGMTEVGA ${ }^{*}$ SCNCFCYICCSCSSA

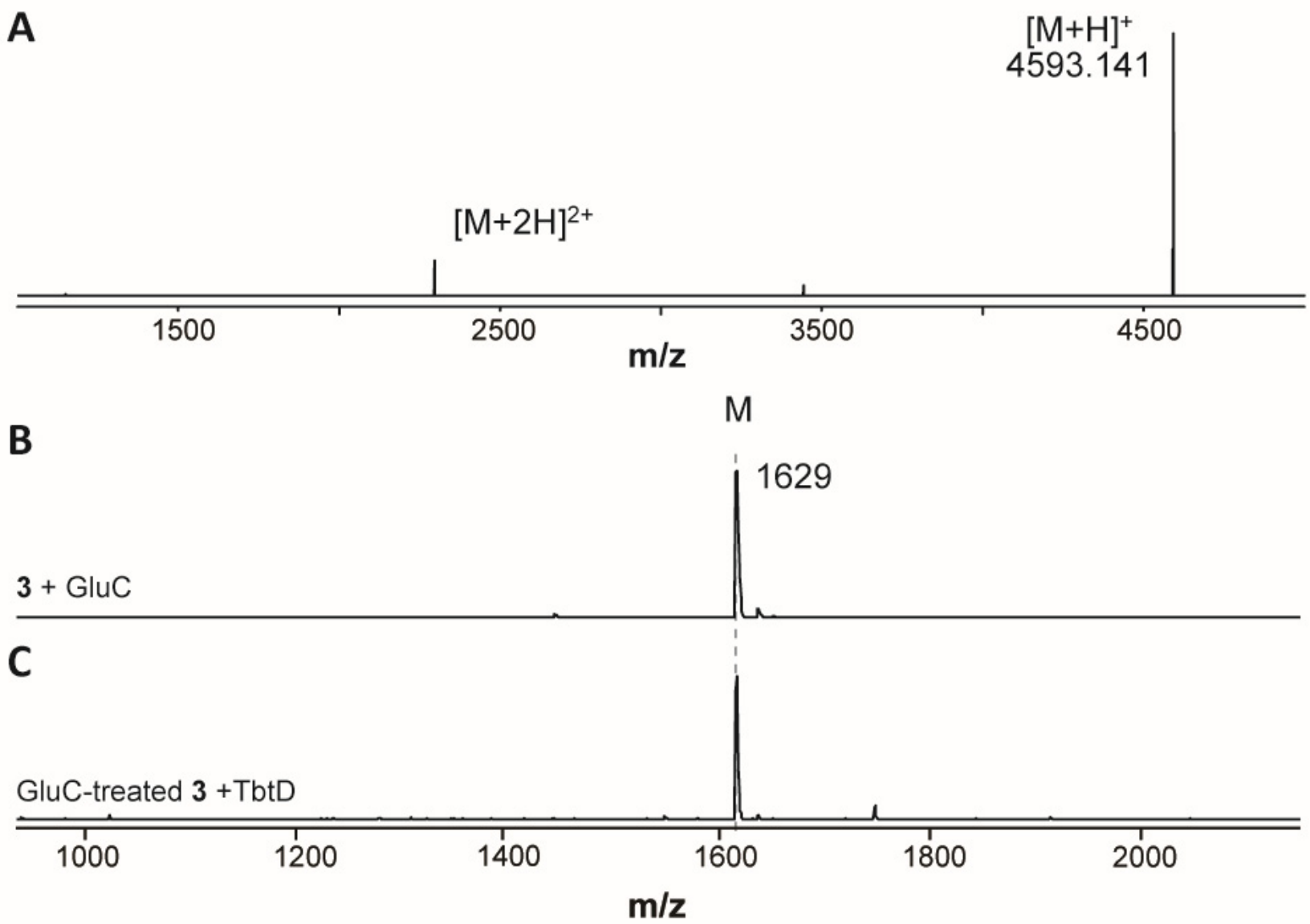


Figure S7: TbtD activity is not enhanced by known leader peptide-binding Tbt proteins. Addition of TbtB or TbtF did not increase the rate of macrocycle formation by TbtD as monitored by UV-Vis spectroscopy at $315 \mathrm{~nm}$ (owing to the generation of the tri-thiazole-substituted pyridine). Both TbtB and TbtF contain RiPP leader peptide Recognition Elements (RREs) ${ }^{9}$ while TbtD does not appear to, despite its activity being leader peptide-dependent (Figure S6).

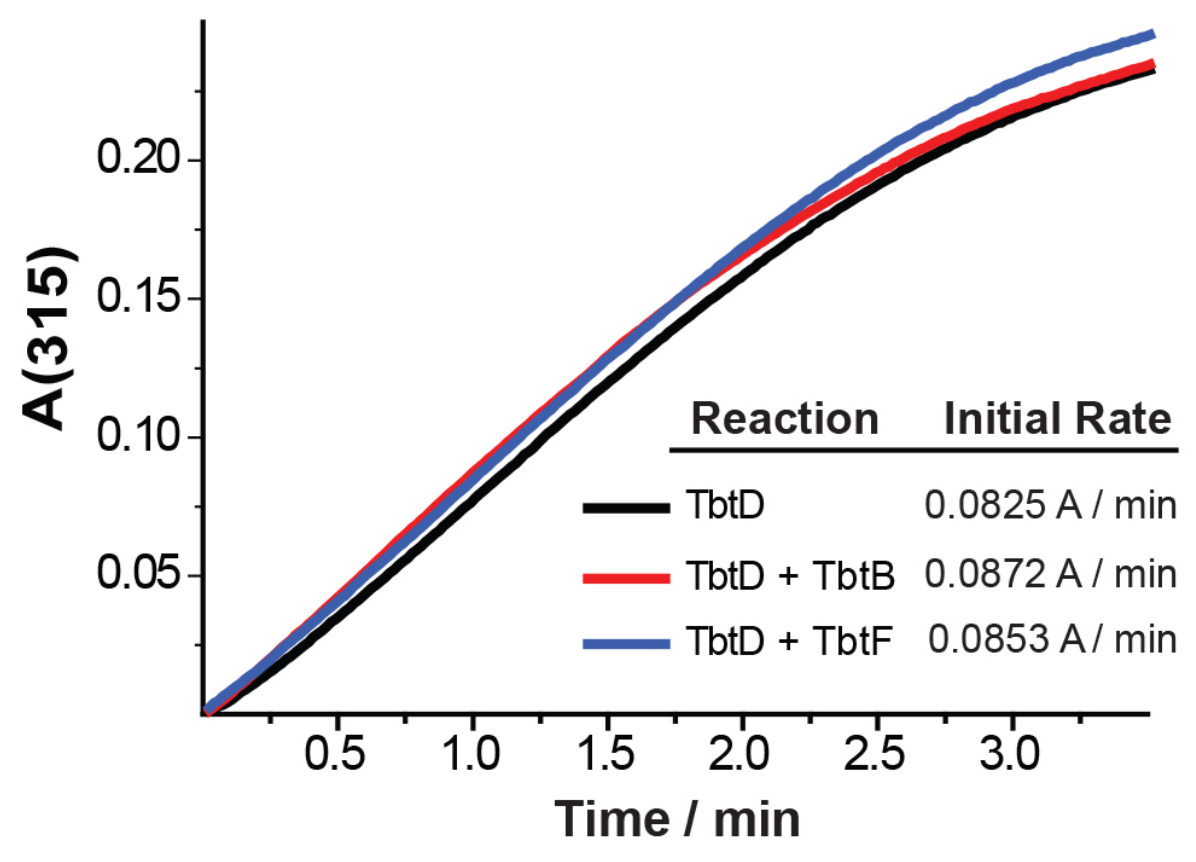


Figure S8: Purity analysis of 4 (macrocyclized thiomuracin GZ). (A) Total ion chromatogram (TIC) monitoring $\mathrm{m} / \mathrm{z}$ 50-2000 of HPLC-purified 4. (B) Extracted ion chromatogram of the $m / 2$ (EIC, $m / z 683.61 \mathrm{Da}$ ) for 4. (C) Analytical HPLC analysis of purified 4 showing UV traces for absorbance at 220 and $315 \mathrm{~nm}$. Hydrolysis of the $C$ terminal dehydroalanine tail of 4 occurs on-column, as indicated by asterisks and verified by re-injection of the 42.4 min eluting product. Including the in situ hydrolysis peaks, the purity was judged to be $\sim 95 \%$, as measured by areaunder-the-curve analysis. Retention times ( $\mathrm{min}$ ) are given for $\mathbf{4}$ and the in situ hydrolysis products.
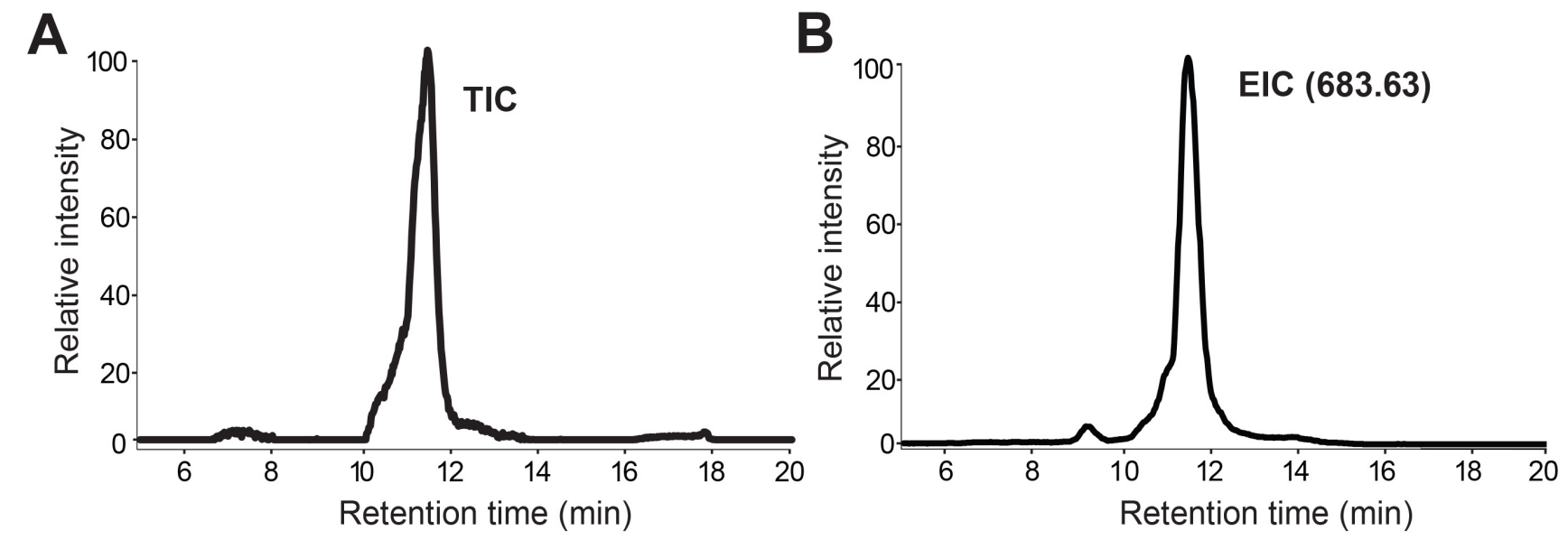

C

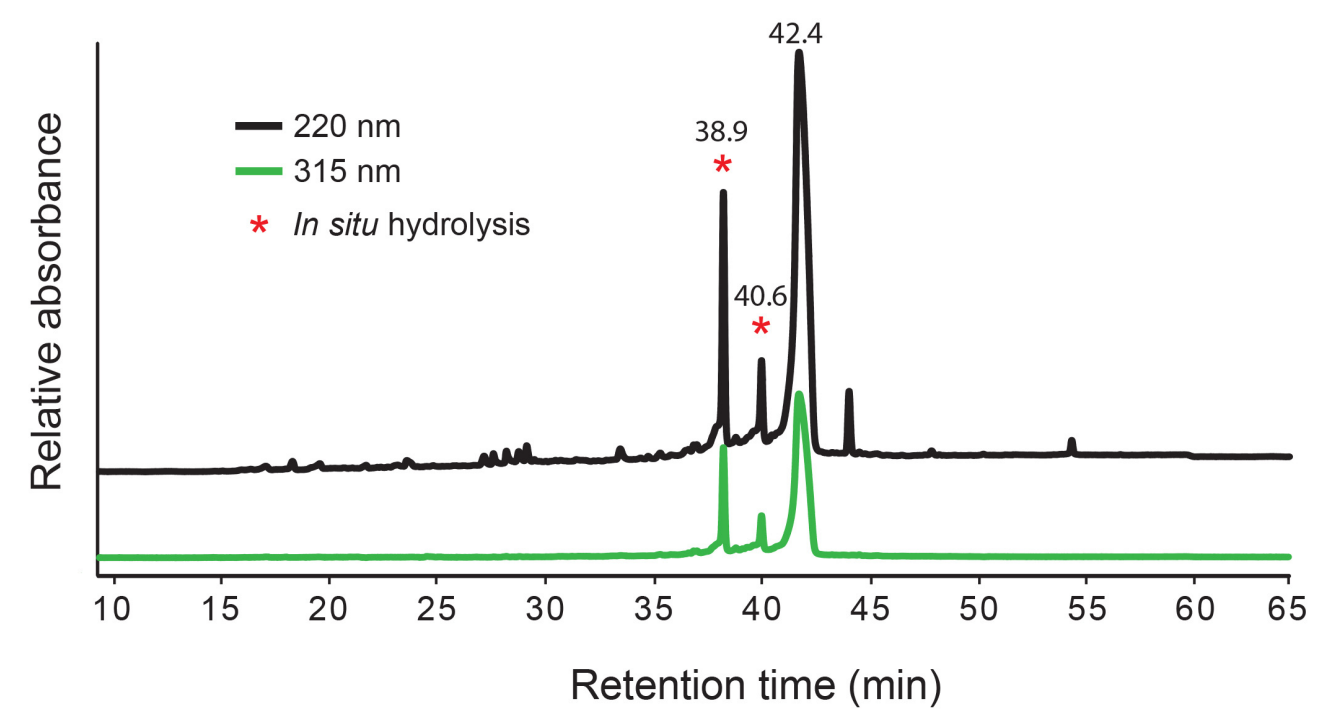


Figure S9: HR-ESI-MS and MS/MS analysis of 4 (macrocyclized product). (A) The molecular formula of the protonated form of 4 was deduced from HR-ESI-MS to be $\mathrm{C}_{61} \mathrm{H}_{56} \mathrm{~N}_{15} \mathrm{O}_{11} \mathrm{~S}_{6}$. (B) Tandem MS of $\mathrm{m} / z 1366$ Da gives a fragmentation pattern consistent with the expected structure.
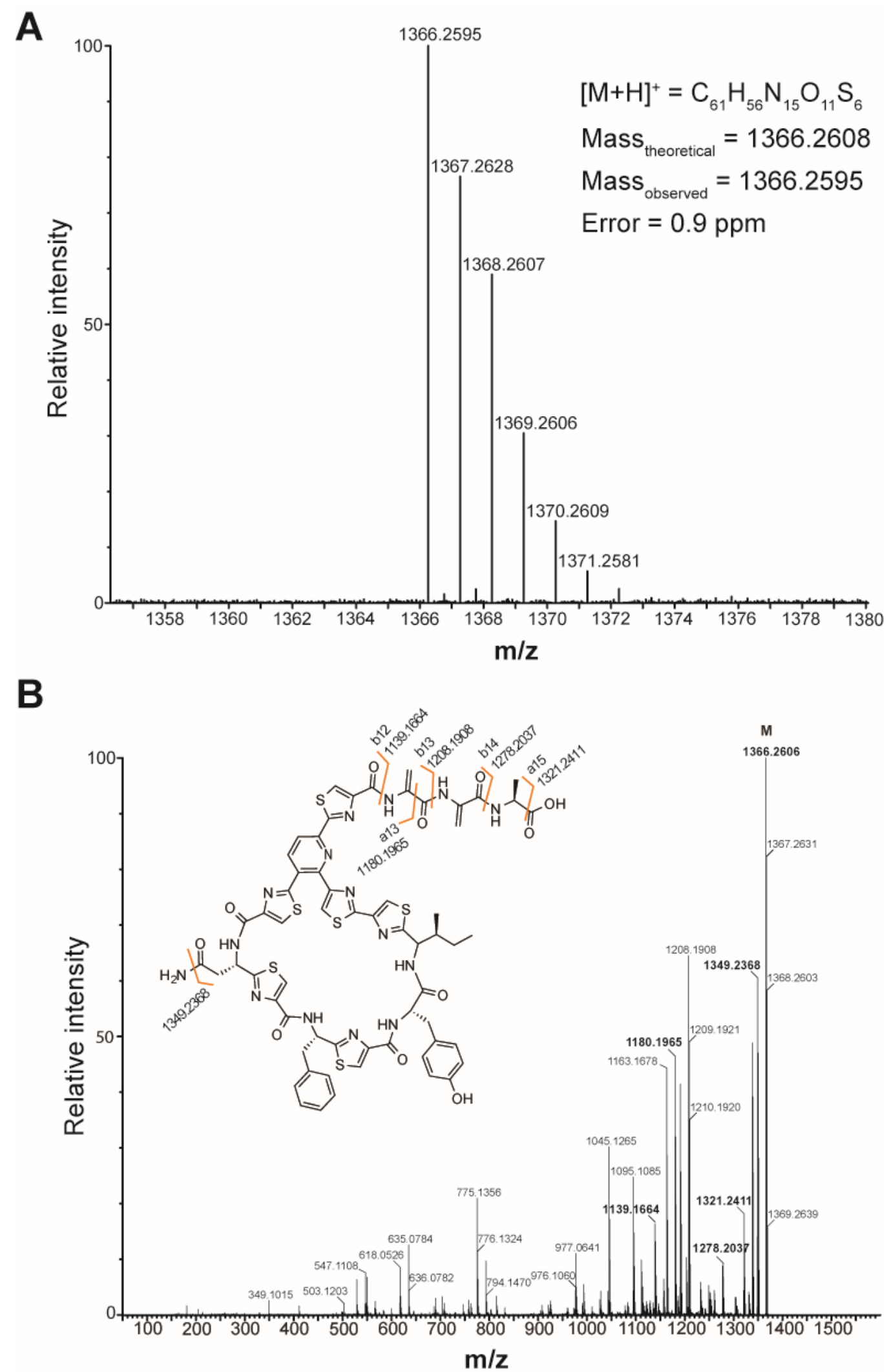
Figure S10: Structurally overlaid assignments for atom numbering and ${ }^{1} \mathrm{H} /{ }^{13} \mathrm{C}$ NMR chemical shifts of 4 .

A

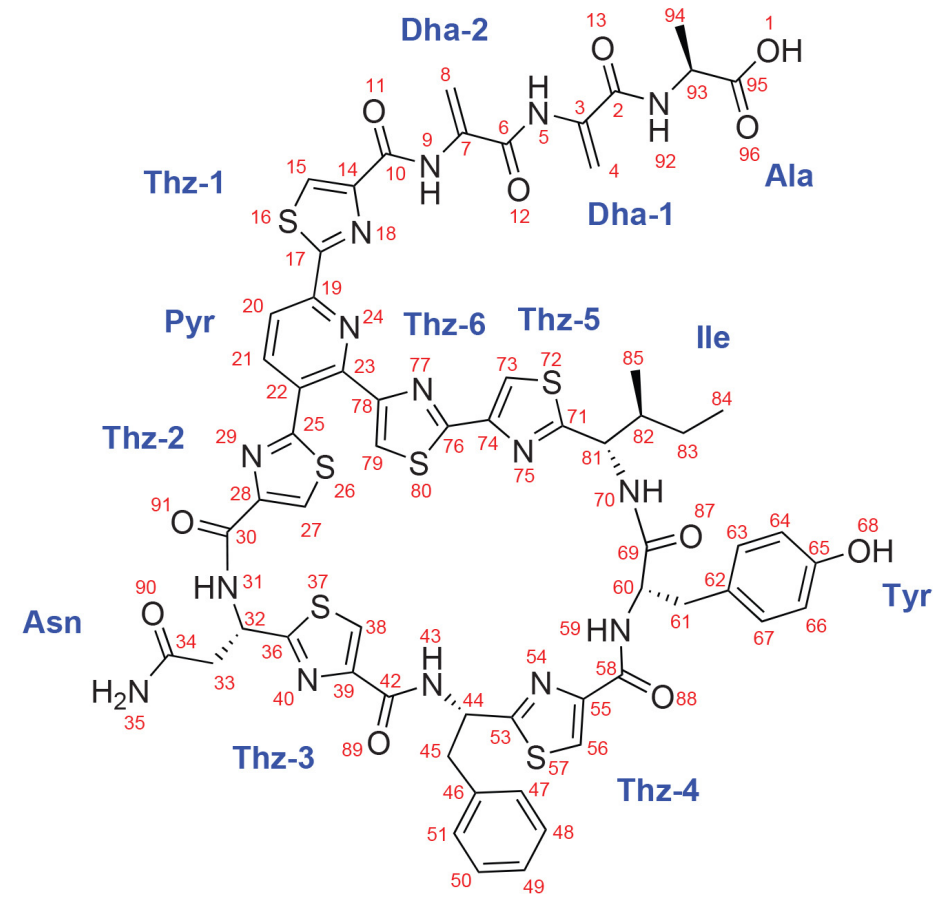

Phe

B

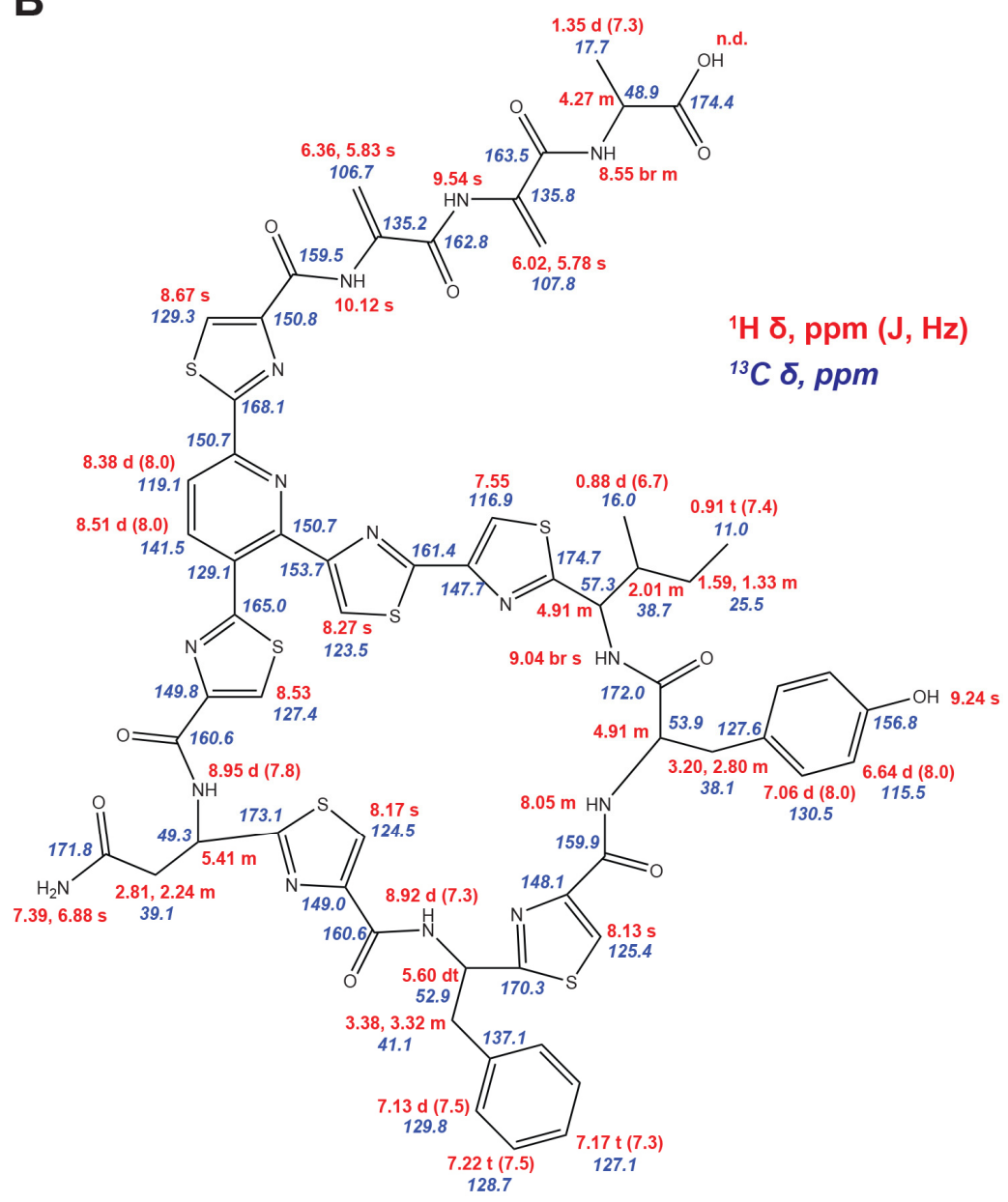


Table S3: ${ }^{1} \mathrm{H}$ and ${ }^{13} \mathrm{C}$ NMR chemical shift assignments of 4 .

${ }^{13} \mathrm{C} \delta \quad{ }^{1} \mathrm{H} \delta \quad \mathrm{m}$ Position Group (ppm) (ppm) (J, Hz) Residue

\begin{tabular}{|c|c|c|c|c|c|}
\hline 92 & $\mathrm{NH}$ & - & 8.55 & br m & Ala \\
\hline 93 & $\mathrm{CH}$ & 48.9 & 4.27 & $\mathrm{~m}$ & \\
\hline 94 & $\mathrm{CH} 3$ & 17.7 & 1.35 & $\mathrm{~d}(7.3)$ & \\
\hline 95 & $C=O$ & 174.4 & - & - & \\
\hline 2 & $C=O$ & 163.5 & - & - & Dha-1 \\
\hline 3 & $C$ & 135.8 & - & - & \\
\hline \multirow[t]{2}{*}{4} & $\mathrm{CH} 2$ & 107.8 & 6.02 & $s$ & \\
\hline & & & 5.78 & $s$ & \\
\hline 5 & $\mathrm{NH}$ & - & 9.54 & $\mathrm{~s}$ & \\
\hline 6 & $C=O$ & 162.8 & - & - & Dha-2 \\
\hline 7 & $C$ & 135.2 & - & - & \\
\hline \multirow[t]{2}{*}{8} & $\mathrm{CH} 2$ & 106.7 & 6.36 & $s$ & \\
\hline & & & 5.83 & $\mathrm{~s}$ & \\
\hline 9 & $\mathrm{NH}$ & - & 10.12 & $\mathrm{~s}$ & \\
\hline 10 & $C=O$ & 159.5 & - & - & Thz-1 \\
\hline 14 & C & 150.8 & - & - & \\
\hline 15 & $\mathrm{CH}$ & 129.3 & 8.67 & $\mathrm{~s}$ & \\
\hline 17 & C & 168.1 & - & - & \\
\hline 19 & C & 150.7 & - & - & Pyr \\
\hline 20 & $\mathrm{CH}$ & 119.1 & 8.38 & $\mathrm{~d}(8.0)$ & \\
\hline 21 & $\mathrm{CH}$ & 141.5 & 8.51 & $\mathrm{~d}(8.0)$ & \\
\hline 22 & C & 128.9 & - & - & \\
\hline 23 & C & 150.7 & - & - & \\
\hline 25 & $C$ & 164.8 & - & - & Thz-2 \\
\hline 27 & $\mathrm{CH}$ & 127.4 & 8.53 & $s$ & \\
\hline 28 & $C$ & 149.8 & - & - & \\
\hline 30 & $C=O$ & 160.6 & - & - & \\
\hline 31 & $\mathrm{NH}$ & - & 8.95 & $\mathrm{~d}(7.8)$ & Asn \\
\hline 32 & $\mathrm{CH}$ & 49.3 & 5.41 & $\mathrm{~m}$ & \\
\hline \multirow[t]{2}{*}{33} & $\mathrm{CH} 2$ & 39.1 & 2.24 & $\mathrm{~m}$ & \\
\hline & & & 2.81 & $\mathrm{~m}$ & \\
\hline 34 & $C=O$ & 171.8 & - & - & \\
\hline \multirow[t]{2}{*}{35} & $\mathrm{NH} 2$ & - & 7.39 & $\mathrm{~s}$ & \\
\hline & & & 6.88 & $s$ & \\
\hline 36 & C & 173.1 & - & - & Thz-3 \\
\hline 38 & $\mathrm{CH}$ & 124.5 & 8.17 & $\mathrm{~s}$ & \\
\hline 39 & $C$ & 149 & - & - & \\
\hline 42 & $C=O$ & 160.6 & - & - & \\
\hline
\end{tabular}

${ }^{13} \mathrm{C} \delta \quad{ }^{1} \mathrm{H} \delta \quad \mathrm{m}$ Position Group (ppm) (ppm) (J, Hz) Residue

\begin{tabular}{|c|c|c|c|c|c|}
\hline 43 & $\mathrm{NH}$ & - & 8.92 & $d(7.3)$ & Phe \\
\hline 44 & $\mathrm{CH}$ & 52.9 & 5.60 & $q(7.0)$ & \\
\hline \multirow[t]{2}{*}{45} & $\mathrm{CH} 2$ & 41.1 & 3.38 & $\mathrm{~m}$ & \\
\hline & & & 3.32 & $\mathrm{~m}$ & \\
\hline 46 & $\mathrm{C}$ & 137.1 & - & - & \\
\hline 47 & $\mathrm{CH}$ & 129.8 & 7.13 & $d(7.5)$ & \\
\hline 48 & $\mathrm{CH}$ & 128.7 & 7.22 & $\mathrm{t}(7.5)$ & \\
\hline 49 & $\mathrm{CH}$ & 127.1 & 7.17 & $\mathrm{t}(7.3)$ & \\
\hline 50 & $\mathrm{CH}$ & 128.7 & 7.22 & $\mathrm{t}(7.5)$ & \\
\hline 51 & $\mathrm{CH}$ & 129.8 & 7.13 & $d(7.5)$ & \\
\hline 53 & $C$ & 170.3 & - & - & Thz-4 \\
\hline 55 & C & 148.1 & - & - & \\
\hline 56 & $\mathrm{CH}$ & 153.7 & 8.13 & s & \\
\hline 58 & $C=O$ & 159.9 & - & - & \\
\hline 59 & $\mathrm{NH}$ & - & 8.05 & $\mathrm{~m}$ & Tyr \\
\hline 60 & $\mathrm{CH}$ & 53.9 & 4.91 & $\mathrm{~m}$ & \\
\hline \multirow[t]{2}{*}{61} & $\mathrm{CH} 2$ & 38.1 & 3.20 & $\mathrm{~m}$ & \\
\hline & & & 2.80 & $\mathrm{~m}$ & \\
\hline 62 & C & 127.6 & - & - & \\
\hline 63 & $\mathrm{CH}$ & 130.5 & 7.06 & $d$ (obs) & \\
\hline 64 & $\mathrm{CH}$ & 115.5 & 6.64 & $d(8.0)$ & \\
\hline 65 & $C$ & 156.8 & - & - & \\
\hline 66 & $\mathrm{CH}$ & 115.5 & 6.64 & $d(8.0)$ & \\
\hline 67 & $\mathrm{CH}$ & 130.5 & 7.06 & $d$ (obs) & \\
\hline 68 & $\mathrm{OH}$ & - & 9.24 & $\mathrm{~s}$ & \\
\hline 69 & $C=O$ & 160 & - & - & \\
\hline 71 & C & 174.7 & - & - & Thz-5 \\
\hline 73 & $\mathrm{CH}$ & 116.9 & 7.55 & s & \\
\hline 74 & $\mathrm{C}$ & 147.7 & - & - & \\
\hline 76 & $C$ & 161.4 & - & - & Thz-6 \\
\hline 78 & $\mathrm{C}$ & 153.7 & - & - & \\
\hline 79 & $\mathrm{CH}$ & 123.5 & 8.27 & $s$ & \\
\hline 70 & $\mathrm{NH}$ & - & 9.04 & br s & Ile \\
\hline 81 & $\mathrm{CH}$ & 57.3 & 4.91 & $\mathrm{~m}$ & \\
\hline 82 & $\mathrm{CH}$ & 38.7 & 2.01 & $\mathrm{~m}$ & \\
\hline \multirow[t]{2}{*}{83} & $\mathrm{CH} 2$ & 25.5 & 1.59 & $\mathrm{~m}$ & \\
\hline & & & 1.33 & $\mathrm{~m}$ & \\
\hline 84 & $\mathrm{CH} 3$ & 11.0 & 0.91 & $\mathrm{t}(7.4)$ & \\
\hline 85 & $\mathrm{CH} 3$ & 16.0 & 0.88 & $d(6.7)$ & \\
\hline
\end{tabular}


Figure S11: ${ }^{1} \mathrm{H}$ NMR $\left(900 \mathrm{MHz}, \mathrm{DMSO}-d_{6}\right)$ spectrum of 4.

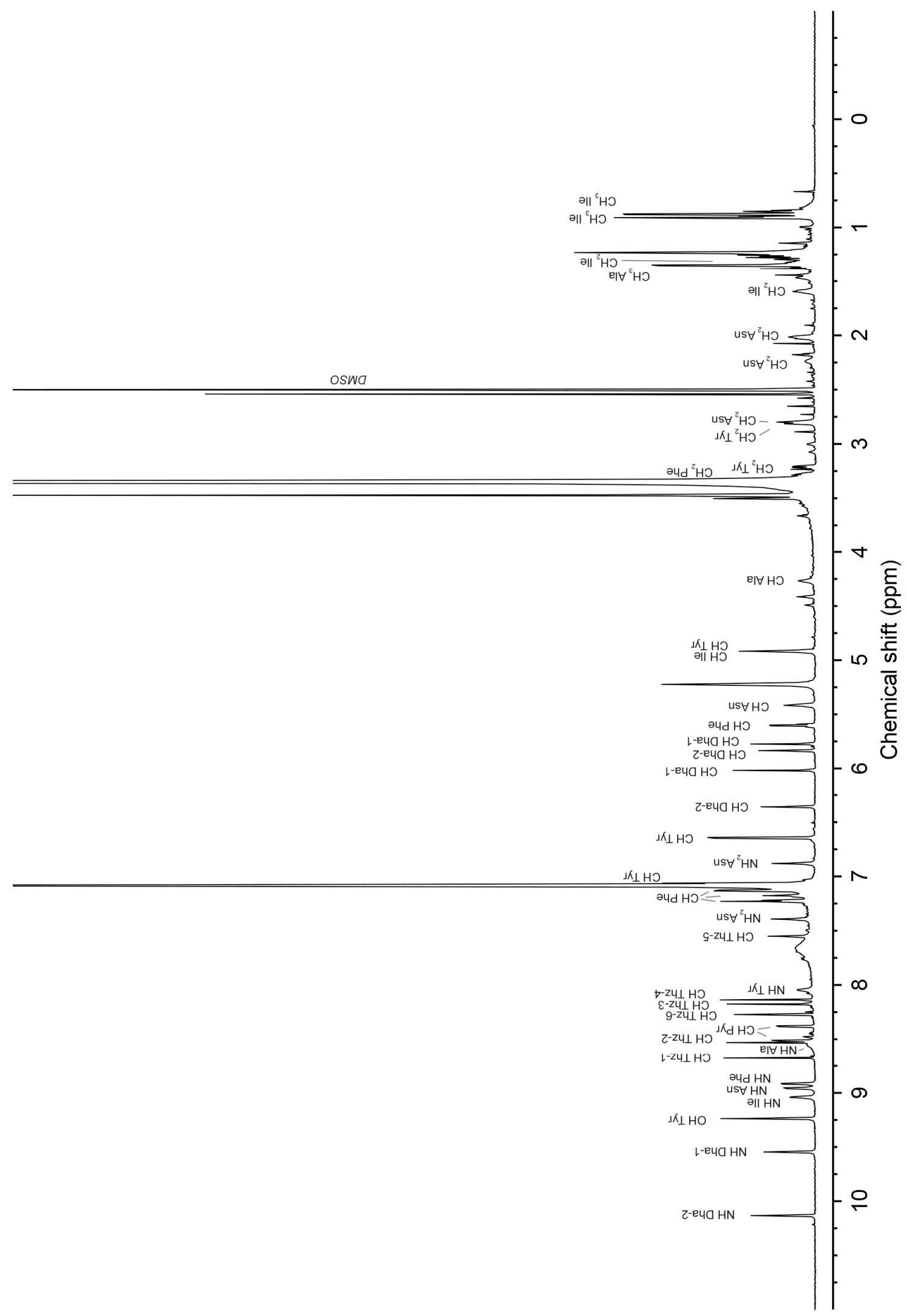


Figure S12: ${ }^{1} \mathrm{H}-{ }^{1} \mathrm{H}$ COSY $\left(900 \mathrm{MHz}, \mathrm{DMSO}-d_{6}\right)$ spectrum of 4.

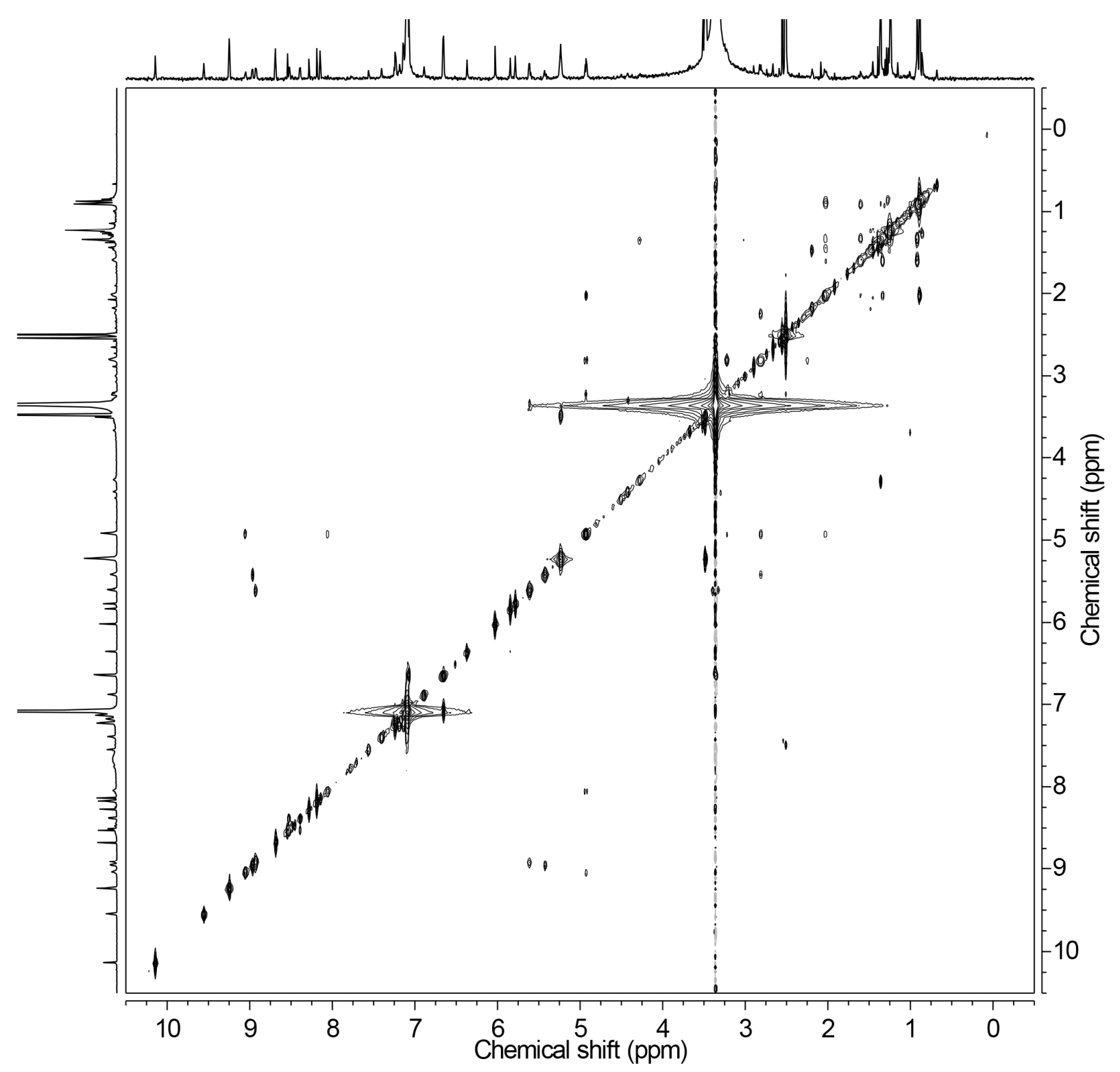


Figure S13: ${ }^{1} \mathrm{H}-{ }^{1} \mathrm{H}$ TOCSY $\left(900 \mathrm{MHz}, \mathrm{DMSO}-d_{6}\right)$ spectrum of 4.

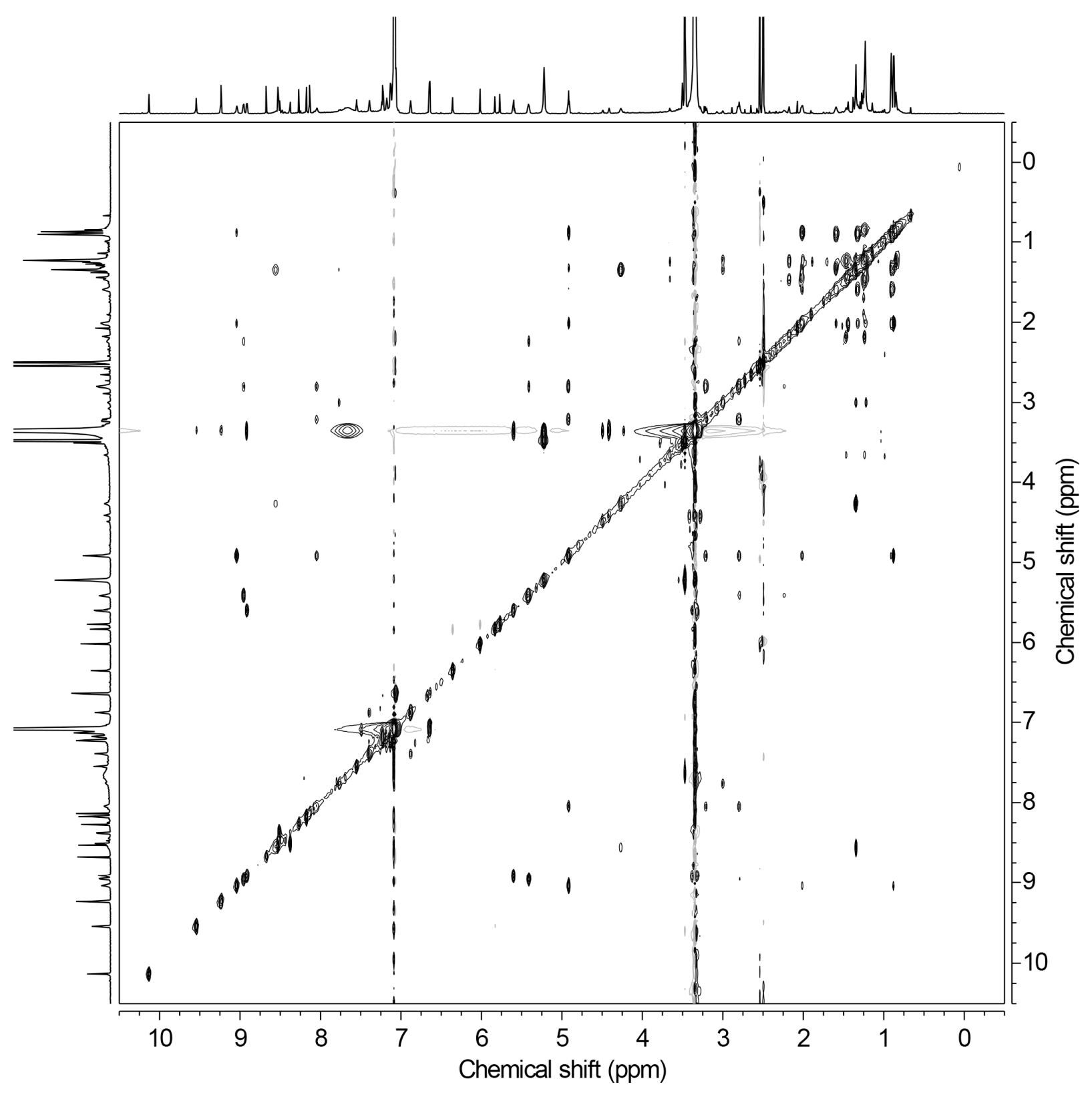


Figure S14: ${ }^{1} \mathrm{H}-{ }^{13} \mathrm{C}$ HMBC (900 MHz, DMSO- $\left.d_{6}\right)$ spectrum of 4.

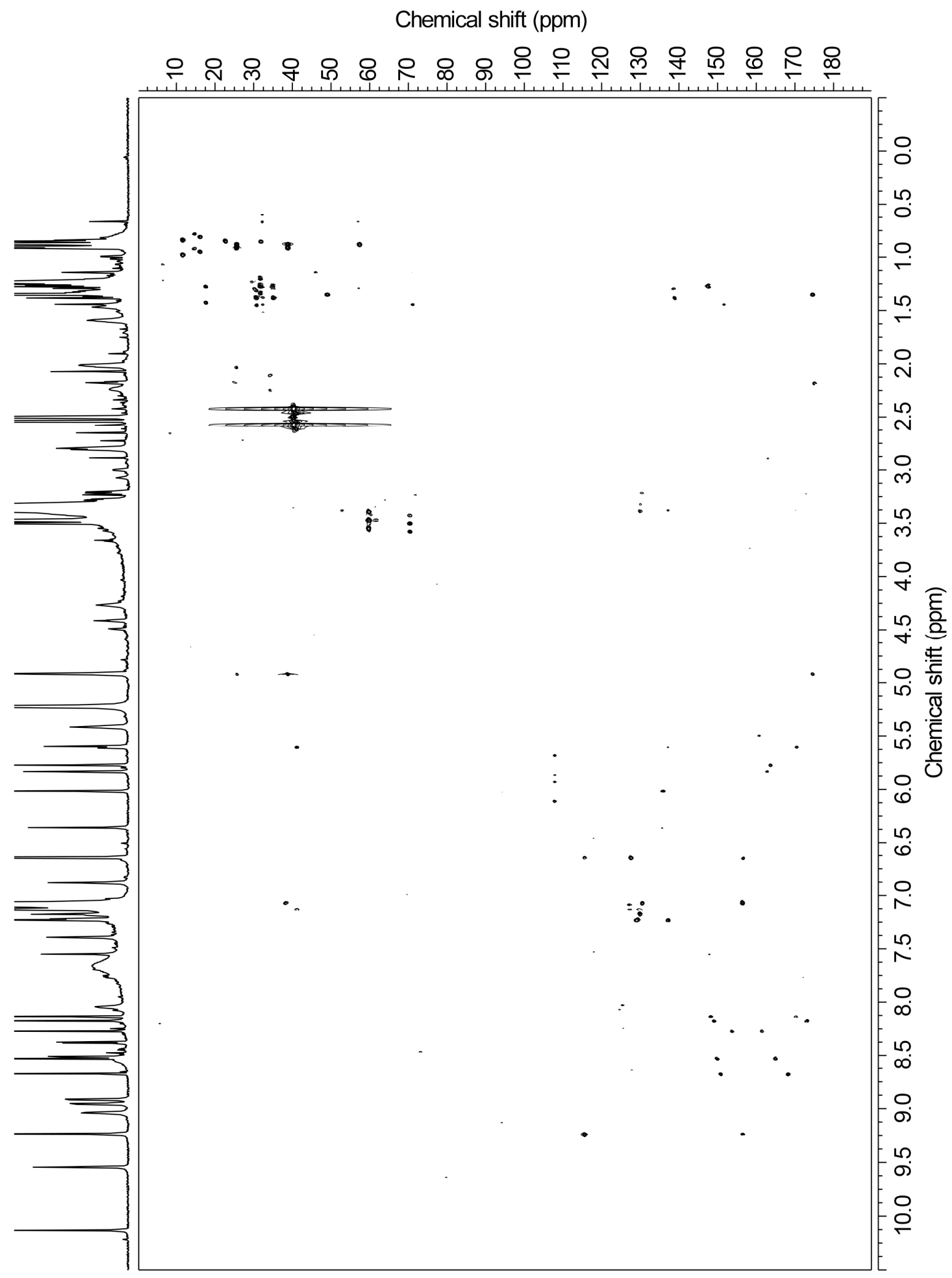


Figure S15: ${ }^{1} \mathrm{H}-{ }^{13} \mathrm{C}$ HSQC (900 MHz, DMSO- $d_{6}$ ) spectrum of 4 (downfield).

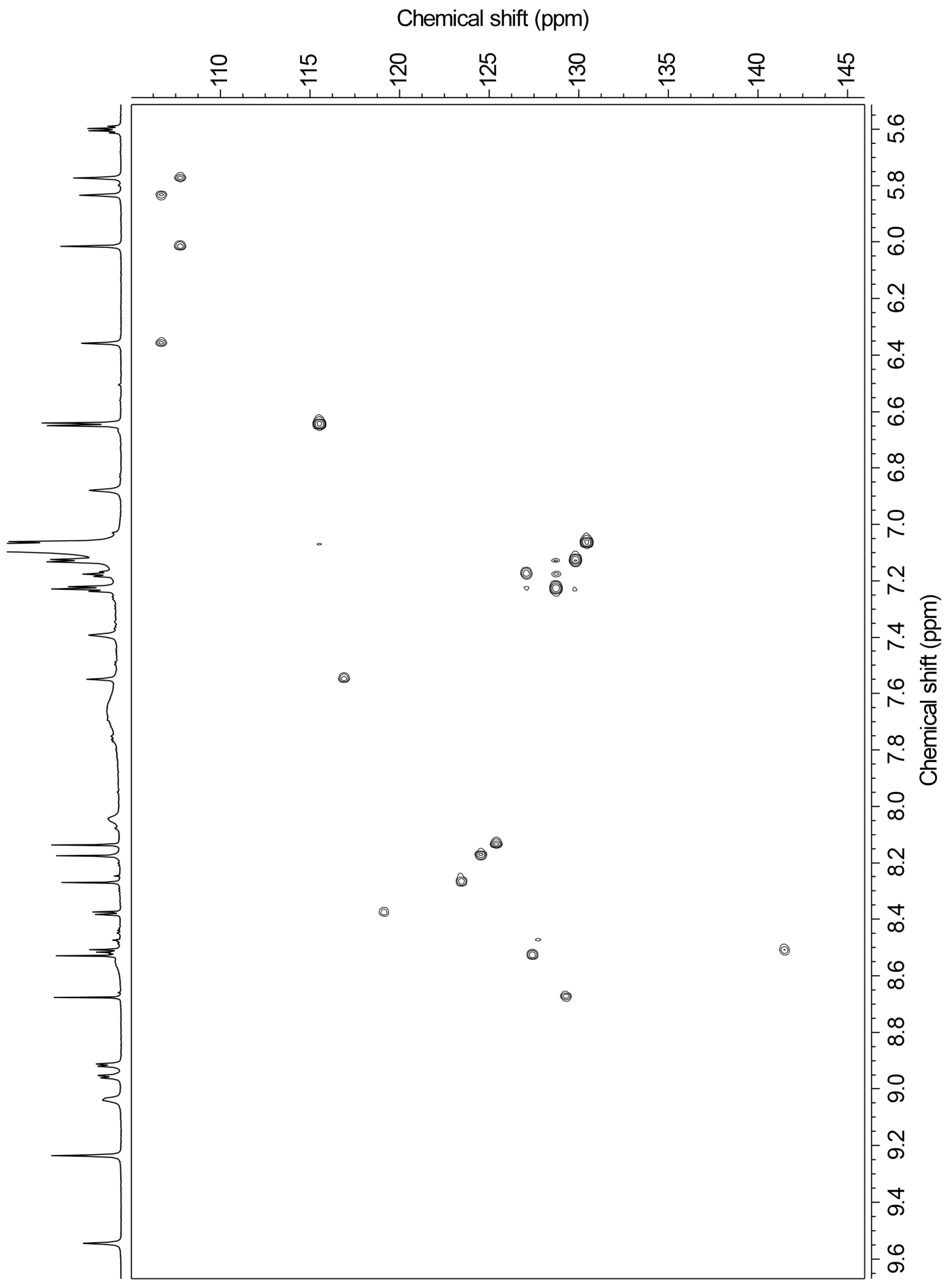


Figure S16: ${ }^{1} \mathrm{H}-{ }^{13} \mathrm{C}$ HSQC $\left(900 \mathrm{MHz}, \mathrm{DMSO}-d_{6}\right)$ spectrum of 4 (upfield).

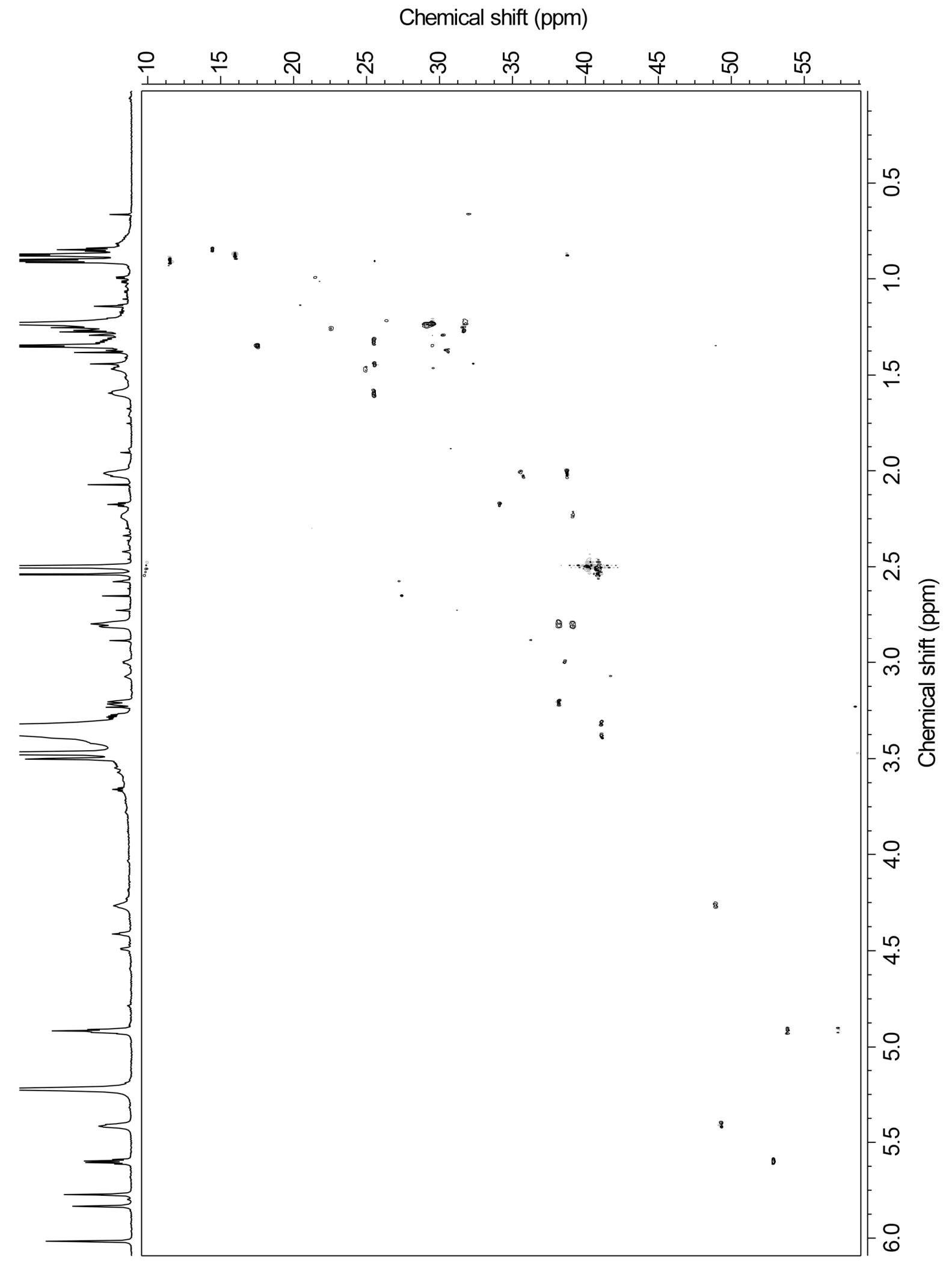


Figure S17: ${ }^{1} \mathrm{H}-{ }^{1} \mathrm{H}$ NOESY $\left(900 \mathrm{MHz}, \mathrm{DMSO}-d_{6}\right)$ spectrum of 4.

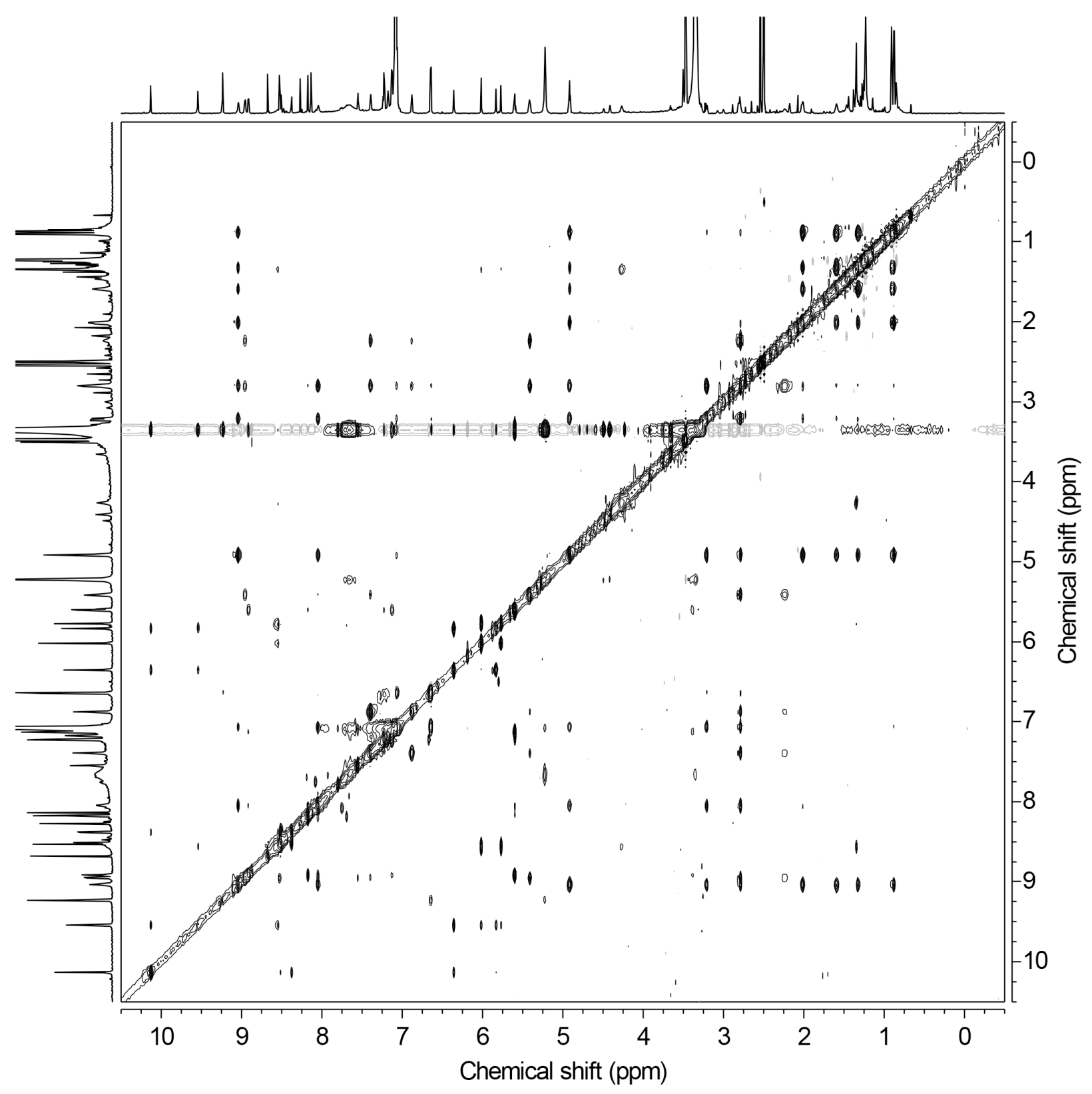


Figure S18: PbtD accepts 3 as a substrate. (A) The amino acid composition of $\mathrm{His}_{6}-\mathrm{TbtA}$ is shown. In peptide 3 the residues shown in blue are thiazoles and in green are dehydroalanines. In the top spectrum, $\mathbf{3}$ is not visible owing to the mass window shown. Reaction of $\mathbf{3}$ with PbtD (homolog of TbtD from the GE2270A biosynthetic gene cluster) shows the formation of $m / z 1366 \mathrm{Da}$ (compare to Figure 2E) and the corresponding sodium adduct. (B) The aligned amino acid sequences of the thiomuracin GZ and GE2270A precursor peptides, labeled TbtA and PbtA, respectively. (C) Structural comparison between thiomuracin GZ and GE2270A. While structurally related, there exist key differences in amino acid composition and post-translational modifications.

His6-TbtA: PHHHHHHSQVDLNDLPMDVFELADSGVAVESLTAGHGMTEVGA*SCNCFCYICCSCSSA

A

3

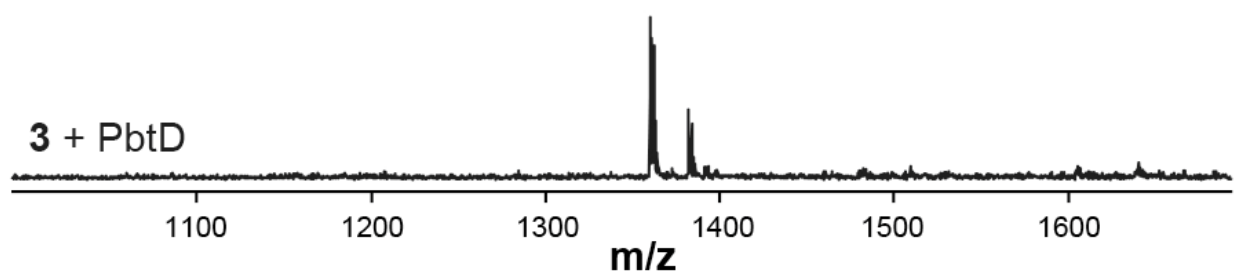

B

TbtA -----MDLNDLPMDVFELADSGVAVESLTAGHGMTEVGASCNCFCYICCSCSSA-

PbtA MSEMELNLNDLPMDVFEMADSGMEVESLTAGHGMPEVGASCNCVCGFCCSCSPSA

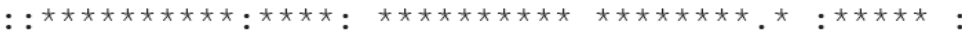

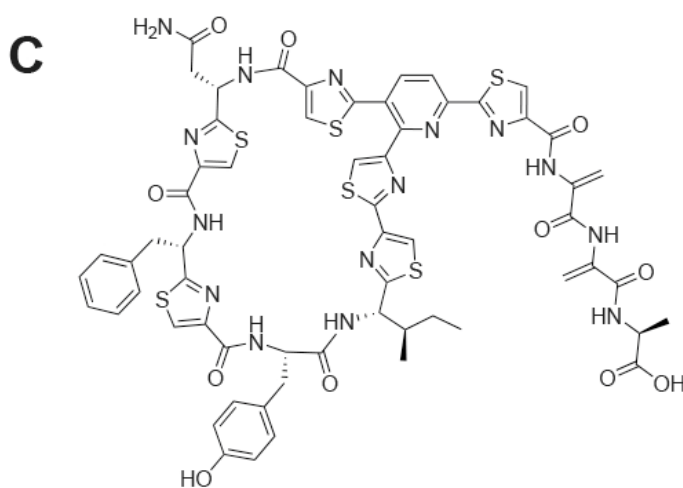

Thiomuracin GZ (4)

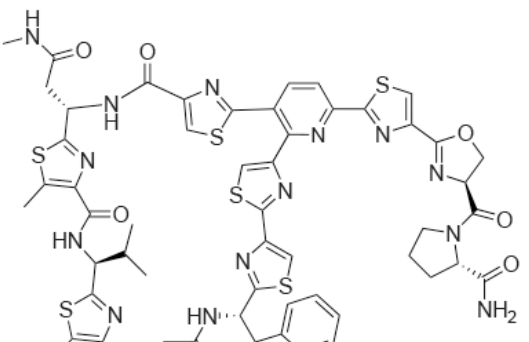

GE2270A 
Figure S19: Sequence alignment of TbtD homologs. GI numbers are given to the left of the alignment (green indicates the sequence from the canonical thiomuracin producer, Nonomuraea sp. Bp3714-39; blue indicates the sequence from the GE2270A producer, Planobispora rosea). Shown in red are residues replaced with alanine for enzymatic activity assessment.

TbtD

gi: 242129450

gi: 552968830

gi: 749676247

gi: 657940957

gi:242129434

Tbt D

gi : 242129450

gi: 552968830

gi: 749676247

gi: 657940957

gi:242129434

TbtD

gi : 242129450

gi : 552968830

gi: 749676247

gi: 657940957

gi:242129434

TbtD

gi : 242129450

gi : 552968830

gi:749676247

gi: 657940957

gi : 242129434

TbtD

gi : 242129450

gi : 552968830

gi: 749676247

gi: 657940957

gi : 242129434

TbtD

gi : 242129450

gi: 552968830

gi: 749676247

gi: 657940957

gi:242129434

TbtD

gi : 242129450

gi: 552968830

gi: 749676247

gi: 657940957

gi : 242129434
MMAAGERWWRFRVDYHAGPM-DDLILDGVRPAFAAFAAQA PMAYFLRHWRRGPHLRIYVS -----MTWTRLRVDYHDGPM-DDL I LDALRPAWHEI-----RGYFLRHWVCGPHLRIFVD -----MTWRRFDVAYHDPDL-DRLILAA-RPLLS---ESPGRSWFQRHWVRGPHLELWFD -- --MEWHS LHVVYYANDK-DRLLLEGVRPLLRRLAPEVAATS FTRHWRLGPHVRI HVK MS GAAATWHS IHVHHHDESTEPALVLHAVRPAFEAVRASVDGAWFGRHWLRGPHLRLNFR -----MSWRRVDVAYHDPDL-DGLILAT-RPLLA---GTPGRGWFQRHWVRGPHLELWFD

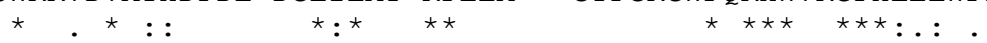

TTREALEAVVRPAIEHVVGGYLRARPSPGMADPSAFLPLHERLAELEGEDGPLMPWS PDN GDATP IV--------RAVERHLAACP SAGTTDPQALLPLHERLAELEGERGPLLPWAPDN HPEPS-----WERVREVLGTHLRAHPSRTRIDPDRLLPQHRRLALAEQ I DEPLLPFYDDN CAPAAVPALVRPAAEE IVGGFLARHP SCTHLVPEDHLDEHRRLAELEEDDRPLLPWRPDN TTADAWASKVRPQVVAVLETYVRDHPSTVRLDEAALAPVHERLAELEMETGPRHPWVPDN AAQPS-----WERIRDVLEPWLRVNPSRARIDRDRLLAQHRHLAAAERIDEPLLPFYADN $: \quad: \quad \star * \quad .: \star \star \star * *$ * $* \star$

TIHAEGERPEPL----TVRDVLLADFYADTTPSVYHALERVRSGASLPTIAFDLVVATAH TVTAEP--PGLD----TELDRFLADFYADTTEAAFDALGRVRAGTPLPGIAFDLVVATAH TLHRAVPRSRVHVLGSAAAEDLFHDFHAAASTAAFDQLDAVVAGESRLGLAFELMIAAAH TIHQAPFERRAHVLGSEEVADLLADFHAATADPAFRMTEAVAGGTPRLGLAFDLMVATAH TVLEQPYDHRLPVLGSMRGSEVLADFLSDTNDLAFAMYEHLQGGGALPVLALDLMWTTAS TLHRAAPRSRAHVLGGPAAEELFHDFHTTASAVAYDELDAVRAGESRLVMALDLMVAAAH

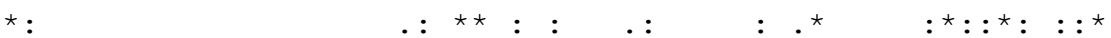

A------LSTGGLPVART SLRSHAEAYLARRS DGVRLRELWRDHYARNREAFTERLIAVA D------LSEGGLPTARTSLRS HAEAYLSRLPGGVR--AKWQAHYERNQEPLTARIKALT A------HAEGGITGGFVSFRSHAEAFLAGAA---GLRERWEAEYRTRAEALRAQVAAVV A------LSGVGITTGFMSERS HAEAFLTTHPEAHRLRPSWDAHYRGHAASLCDRVGRVT VAAVPFDEHSAP IARGFLSLRS HADAFLSRTHDPAAYRAAFDERYRRQADALTTRLRTVE A------HAEGGVRGGFVSFRSHAEAFLASAP---GLRERWDAEYAARAGALRARITAVV $: \quad{ }^{*}:{ }^{*}{ }^{*}:{ }^{*}:{ }^{*}: \quad{ }^{*} . \quad: \quad:: \quad:$

SSAE-----SAENGAHLPHVREWVRRLRPIRERARALLESGELTLEYASPAEGARDLPSL GAG--------------EPGAWLRT IRATRDRGRTLIDEGRLSLGYAT--DGPSTRPPL TG------------ TPRGRAWTGLLDGFAGRGDELIASGALTVEPASPT-AAAEP--GALD-----DGS--GGVGFTGEWTELMRSFRRRGRALLAEGRLPSETAFAPGADGRPPPL SVLSAEPGS PEAADATVPFVREWAEAVRRCQRAAYPLLASGELTLSGAGRA----PRLPT AG------------I IPRGRAWAGLLDRFADRGDELIASGALLVEPAGPD-AVARP---

AEVSAFHRELESRPEW-ARLRDSPAFGAYRLVINCTYLHLTRLGLTPHQRFLVCHLAADA AAVSPFHRNLETDERW-LALKDTPAFAAYRLALNCAYLHLTRLGLTPDQRFLICHLAASA --DTEFHRALRANRTWHDEVLRSPS FRRYRLLLNLTYLQMSRLGVTAVQRSLLCHFAASA AGASPYHRAAYRNPAV-MASMTAEWFVLYRLMLNYTYLFMTQLGVTPVERYLLCHLTAHA RQLSDFHRLLQTDHGHDGFLRDDHWFASFRLVVNYLYVHLNRLGLKPVDRGLLCHLAAGT --DTAFHRALRGNRTWHEEVLRSAPFRRYRLLLNLTYLQLSRLGVNAVQRALLCHFAASA

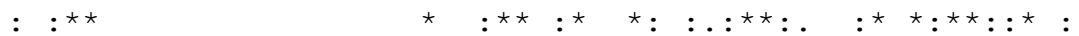

AADVYGIAAHEEVATR-----------------------

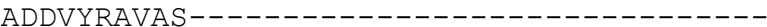

VEEEYGVSAIEIAVGGM----------------------

VEELYGVSAMEQITRAPPEPVLDAGGEPR------------

VETVHGVTAEDAFRRHVVSASP-TEDTPEWSRLSKEWAEGR

VEQEYGVSAIEIAMGGA----------------------- 
Table S4: Yield and activity of alanine-substituted TbtD proteins. Twenty-three positions of TbtD were targeted for alanine replacement by site-directed mutagenesis using the alignment of Fig. S18. These proteins were expressed in $E$. coli as MBP fusions, purified to homogeneity (Fig. S1), then assayed for enzymatic activity using a MALDITOF-MS endpoint assay. In condition A, high concentrations of enzyme and substrate were used to assess the formation of $\mathbf{4}$. In condition $\mathbf{B}$, both the enzyme and substrate concentration were lowered. Precise conditions follow for A: HEPES pH $7.5(100 \mathrm{mM}), \mathrm{MgCl}_{2}(5 \mathrm{mM}), \mathrm{KCl}(150 \mathrm{mM})$, ATP $(5 \mathrm{mM}), 2$ (hexazole, $\left.100 \mu \mathrm{M}\right)$, T. bispora tRNA $^{\text {Glu }}(10 \mu \mathrm{M})$, E. coli GluRS $(10 \mu \mathrm{M})$, TbtB $(5 \mu \mathrm{M})$, TbtC $(5 \mu \mathrm{M})$, MBP-TbtD $(5 \mu \mathrm{M})$, and TIPP $(0.02 \mathrm{U} / \mu \mathrm{L})$. The reaction was allowed to proceed at $30^{\circ} \mathrm{C}$ for $4 \mathrm{~h}$. Conditions for $\mathbf{B}$ were identical to $\mathbf{A}$ except for the following changes: 2 (hexazole, $15 \mu \mathrm{M}$; lowered 6.67-fold), T. bispora tRNA ${ }^{\text {Glu }}(10 \mu \mathrm{M})$, E. coli GluRS $(10 \mu \mathrm{M})$, TbtB $(5$ $\mu \mathrm{M})$, TbtC $(5 \mu \mathrm{M}), \mathrm{MBP}-\mathrm{TbtD}\left(1 \mu \mathrm{M}\right.$; lowered 5-fold), and TIPP $\left(0.02 \mathrm{U}_{\mu \mathrm{L}^{-1}}\right)$. The reaction was allowed to proceed at $30{ }^{\circ} \mathrm{C}$ for $4 \mathrm{~h} .+++$ indicates enzyme activity roughly equal to wild-type (full conversion to product); ++ indicates modestly reduced enzyme activity ( $\sim 50 \%$ conversion to product); + indicates severely reduced enzyme activity (low but detectable product formation); - indicates no detectable enzyme activity (no observed product formation). For a more quantitative analysis of the proteins that displayed reduced activity, see Table S5. Isolated protein yields are given as a crude measure for protein stability (structurally destabilizing substitutions typically give lower yields and visible degradation by SDS-PAGE).

\begin{tabular}{|c|c|c|c|}
\hline $\begin{array}{c}\text { TbtD } \\
\text { Protein }\end{array}$ & $\begin{array}{c}\text { Protein } \\
\text { Yield (mg/L) }\end{array}$ & $\begin{array}{c}\text { Relative Product } \\
\text { (condition A) }\end{array}$ & $\begin{array}{c}\text { Relative Product } \\
\text { (condition B) }\end{array}$ \\
\hline Wild-type & 14 & +++ & +++ \\
\hline H46A & 8 & +++ & ++ \\
\hline W47A & 17 & +++ & +++ \\
\hline R49A & 8 & +++ & +++ \\
\hline H52A & 8 & +++ & +++ \\
\hline R54A & 21 & +++ & +++ \\
\hline S85A & 13 & +++ & +++ \\
\hline H98A & 8 & +++ & ++ \\
\hline E105A & 11 & +++ & +++ \\
\hline N118A & 8 & +++ & +++ \\
\hline T144A & 13 & +++ & ++ \\
\hline R189A & 11 & +++ & ++ \\
\hline S190A & 8 & +++ & ++ \\
\hline H191A & 4 & +++ & +++ \\
\hline E193A & 11 & +++ & ++ \\
\hline S287A & 13 & +++ & ++ \\
\hline H290A & 10 & +++ & ++ \\
\hline R291A & 10 & +++ & ++ \\
\hline N316A & 13 & ++ & ++ \\
\hline Y319A & 11 & ++ & ++ \\
\hline R324A & 10 & ++ & ++ \\
\hline R332A & 16 & ++ & ++ \\
\hline C336A & 10 & ++ & ++ \\
\hline H337A & 16 & ++ & ++ \\
\hline
\end{tabular}


Table S5: Enzymatic activity of selected TbtD mutant proteins. Seven TbtD mutants were selected based on phenotype in the end-point MALDI-TOF MS assay (Table S4). Conversion efficiency was monitored by comparing EIC intensity to that of wild-type at 90 min (i.e., fully converted). As the wild-type TbtD performed the reaction much faster than the selected mutants, $200 \mathrm{nM}$ enzyme was used for the assay compared to $1 \mu \mathrm{M}$ for the mutant enzymes. All experiments were performed in duplicate. ND = not detected.

\begin{tabular}{|c|c|c|c|}
\hline TbtD protein & $\begin{array}{c}\text { \% conversion } \\
\mathbf{3 0} \mathbf{~ m i n}\end{array}$ & $\begin{array}{c}\text { \% conversion } \\
\mathbf{6 0} \mathbf{~} \mathbf{n}\end{array}$ & $\begin{array}{c}\text { \% conversion } \\
\mathbf{9 0} \mathbf{~} \text { in }\end{array}$ \\
\hline Wild-type & $77 \pm 2$ & $87 \pm 1$ & 100 \\
\hline W47A & $36 \pm 3$ & $55 \pm 2$ & $64 \pm 1$ \\
\hline E105A & $25 \pm 4$ & $47 \pm 7$ & $54 \pm 11$ \\
\hline H191A & $36 \pm 3$ & $57 \pm 1$ & $67 \pm 4$ \\
\hline S287A & $14 \pm 4$ & $23 \pm 1$ & $39 \pm 11$ \\
\hline H290A & $14 \pm 3$ & $33 \pm 11$ & $42 \pm 4$ \\
\hline Y319A & $2 \pm 1$ & $5.4 \pm 3.7$ & $11 \pm 1$ \\
\hline R332A & ND & ND & ND \\
\hline
\end{tabular}




\section{Supporting References}

(1) Sherlin, L. D.; Bullock, T. L.; Nissan, T. A.; Perona, J. J.; Lariviere, F. J.; Uhlenbeck, O. C.; Scaringe, S. A. RNA 2001, 7, 1671.

(2) Morris, R. P.; Leeds, J. A.; Naegeli, H. U.; Oberer, L.; Memmert, K.; Weber, E.; Lamarche, M. J.; Parker, C. N.; Burrer, N.; Esterow, S.; Hein, A. E.; Schmitt, E. K.; Krastel, P. J. Am. Chem. Soc. 2009, 5946.

(3) Geoghegan, K. F.; Dixon, H. B.; Rosner, P. J.; Hoth, L. R.; Lanzetti, A. J.; Borzilleri, K. A.; Marr, E. S.; Pezzullo, L. H.; Martin, L. B.; LeMotte, P. K.; McColl, A. S.; Kamath, A. V.; Stroh, J. G. Anal. Biochem. 1999, 267, 169.

(4) Gibson, D. G.; Young, L.; Chuang, R. Y.; Venter, J. C.; Hutchison, C. A., 3rd; Smith, H. O. Nat. Methods 2009, 6, 343.

(5) Li, B.; Cooper, L. E.; van der Donk, W. A. Methods Enzymol. 2009, 458, 533.

(6) Garg, N.; Salazar-Ocampo, L. M.; van der Donk, W. A. Proc. Natl. Acad. Sci. U. S. A. 2013, 110, 7258.

(7) Rio, D. C.; Ares, M. J.; Hannon, G. J.; Nilsen, T. W. RNA: A Laboratory Manual; Cold Spring Harbor Laboratory Press, 2011.

(8) Walker, S. E.; Fredrick, K. Methods 2008, 44, 81.

(9) Burkhart, B. J.; Hudson, G. A.; Dunbar, K. L.; Mitchell, D. A. Nat. Chem. Biol. 2015, 11, 564. 